\title{
Overview and reassessment of noise budget of starshade exoplanet imaging
}

\author{
Renyu Hu®, a,b,* Doug Lisman, ${ }^{a}$ Stuart Shaklan $\odot,{ }^{\text {a }}$ Stefan Martin $\odot,{ }^{a}$ \\ Phil Willems $\odot,{ }^{a}$ and Kendra Short ${ }^{a}$ \\ a Jet Propulsion Laboratory, California Institute of Technology, Pasadena, California, \\ United States \\ ${ }^{\mathrm{b}}$ California Institute of Technology, Division of Geological and Planetary Sciences, \\ Pasadena, California, United States
}

\begin{abstract}
High-contrast imaging enabled by a starshade in formation flight with a space telescope can provide a near-term pathway to search for and characterize temperate and small planets of nearby stars. NASA's Starshade Technology Development Activity to TRL5 (S5) is rapidly maturing the required technologies to the point at which starshades could be integrated into potential future missions. We reappraise the noise budget of starshade-enabled exoplanet imaging to incorporate the experimentally demonstrated optical performance of the starshade and its optical edge. Our analyses of stray light sources-including the leakage through micrometeoroid damage and the reflection of bright celestial bodies-indicate that sunlight scattered by the optical edge (i.e., the solar glint) is by far the dominant stray light. With telescope and observation parameters that approximately correspond to Starshade Rendezvous with Roman and Habitable Exoplanet Observatory (HabEx), we find that the dominating noise source is exozodiacal light for characterizing a temperate and Earth-sized planet around Sun-like and earlier stars and the solar glint for later-type stars. Further, reducing the brightness of solar glint by a factor of 10 with a coating would prevent it from becoming the dominant noise for both Roman and HabEx. With an instrument contrast of $10^{-10}$, the residual starlight is not a dominant noise, and increasing the contrast level by a factor 10 does not lead to any appreciable change in the expected science performance. If unbiased calibration of the background to the photon-noise limit can be achieved, Starshade Rendezvous with Roman could provide nearly photon-limited spectroscopy of temperate and Earth-sized planets of F, G, and K stars $<4$ parsecs away, and HabEx could extend this capability to many more stars $<8$ parsecs. Larger rocky planets around stars $<8$ parsecs would be within the reach of Roman. To achieve these capabilities, the exozodiacal light may need to be calibrated to a precision better than $2 \%$ and the solar glint to better than 5\%. Our analysis shows that the expected temporal variability of the solar glint is unlikely to hinder the calibration, and the main challenge for background calibration likely comes from the unsmooth spatial distribution of exozodiacal dust in some stars. Taken together, these results validate the optical noise budget and technology milestones adopted by S5 against key science objectives and inform the priorities of future technology developments and science and industry community partnerships. (C) The Authors. Published by SPIE under a Creative Commons Attribution 4.0 Unported License. Distribution or reproduction of this work in whole or in part requires full attribution of the original publication, including its DOI. [DOI: 10.1117/1.JATIS.7.2.021205]
\end{abstract}

Keywords: starshade; high-contrast imaging; exoplanet; stray light; Roman space telescope; Habitable exoplanet observatory.

Paper 20107SS received Jul. 30, 2020; accepted for publication Dec. 1, 2020; published online Jan. 8, 2021.

\section{Introduction}

Direct imaging of exoplanets from space holds promise for writing a new chapter in astronomy and planetary science. With most of the exoplanets discovered to date in tightly bound orbits of their host stars, and thus uninhabitable unless the stars are much fainter than the Sun, direct

*Address all correspondence to Renyu Hu, renyu.hu@jpl.nasa.gov 
imaging would detect planets in the habitable zones $(\mathrm{HZs})^{1}$ of more Sun-like stars. If some of these planets are small and predominantly rocky in composition, they may have environments hospitable for life. One of the primary goals of exoplanet direct imaging is to search for temperate and small planets of nearby stars and study the chemical composition of their atmospheres with spectroscopy.

A starshade working in tandem with a space telescope provides one of the best near-term opportunities to achieve this goal. Starshade is an external occulter flown along the line of sight from a telescope to a target star. With the shape designed to mimic the optical effects of an optimally apodized screen, a starshade can create a "deep shadow" where the starlight is suppressed by $10^{10}{ }^{2,3}$ The telescope kept in this shadow would be able to detect planets and disks around the star at very high contrast. A starshade that prevents the starlight from entering the telescope would allow many simplifications of the telescope optics. For example, precise wavefront control would not be necessary, which also reduces the number of reflections before feeding the light to a detector and thus increases the optical throughput of the instrument. The costs of these benefits are the added complexity of formation flying and the complications involved in launching and deploying the large and optically precise starshade.

Two advanced mission concepts to discover Earth-like planets in the HZs of Sun-like stars being considered at NASA will use starshades as one of the starlight suppression techniques. The Roman Space Telescope ${ }^{4,5}$ will be capable of collecting starlight reflected by large exoplanets with its coronagraph instrument. The Starshade Rendezvous Probe, ${ }^{6}$ an advanced mission concept, would further enable Roman to search for Earth-sized planets in the HZs of 10 nearby stars, with the possibility of obtaining their limited-bandwidth spectra at a moderate resolution ( 70). The Habitable Exoplanet Observatory $\left(\mathrm{HabEx}^{7}{ }^{7}\right.$ ), a concept of a 4-m space telescope with a starshade, has the main objective of searching for Earth-sized planets in a larger stellar sample and obtaining their spectra in a wider band and at a higher resolution. Starshade Rendezvous with Roman and HabEx, both with a starshade, would have the spectral characterization of small planets in the HZs of nearby stars as the key and probably limiting science objective.

To enable these potential exoplanet science missions, NASA's Exoplanet Exploration Program (ExEP) is executing a directed and focused activity, the Starshade Technology Development Activity to TRL5 (S5). For S5, TRL5 is defined as demonstrating critical performance in relevant environments at the subsystem level with medium fidelity prototypes. The technology development plan of $\mathrm{S}^{8}$ adopts formation flying and observation scenarios of the Rendezvous and HabEx mission concepts as the baseline. Completion of S5 will bring starshade technologies to TRL5 for both concepts. Specifically, S5 includes experiments and analyses to demonstrate small-scale starshade masks that could reach $10^{-10}$ instrument contrast at the inner working angle (IWA) at a flight-like Fresnel number, to develop an optical edge for the starshade petals that would limit scattered sunlight (i.e., solar glint) to acceptable levels, to demonstrate the ability to sense the lateral offset between the starshade and the telescope to an accuracy of $30 \mathrm{~cm}$, and to demonstrate the ability to design and manufacture the starshade mechanical elements that meet the contrast requirement. Together with S5, ExEP has chartered a Science and Industry Partnership to engage the broader science and technology communities during the execution of the S5 activity.

Given the completion of most of S5's technology milestones on instrument contrast, ${ }^{9,10}$ solar glint, ${ }^{11}$ and formation flying, ${ }^{12}$ we are motivated to revisit the noise budget of starshades' application in exoplanet imaging. Although estimates of exoplanet yields from starshade-assisted imaging have been published, ${ }^{13-15}$ these works have not mapped the technology progress to science performance with the level of detail of this paper. In particular, previous works have not explicitly included solar glint in their noise budget. The purpose of this paper is to update the expected performance of starshade-enabled exoplanet imaging in light of new constraints on starlight and stray light suppression resulting from S5 work and to assess the noise budget of the spectral characterization of temperate and small planets of nearby stars. We focus on the performance parameters that are directly related to exoplanet imaging and defer the assessment on mechanical precision tolerance and stability - which controls the instrument contrast - to an error budget analysis ${ }^{16}$ and the S5 technology development plan. We focus on revealing the dominating noise term under a wide range of realistic planet scenarios to guide the priorities of future development. We first evaluate the science performance on the assumption that the 
background can be calibrated to the photon-noise limit and then discuss the impact of temporal and spatial variability on background calibration.

The reminder of this paper is organized as follows. We first describe the model used to derive the $S / N$ of a starshade-enabled exoplanet observation based on performance parameters in Sec. 2. In Sec. 3, we discuss a range of stray light sources that may enter the telescope and are not included as a potentially dominant term in the noise budget. Section 4 presents the expected $S / N$ for observing nearby planetary systems with the current performance demonstrated by S5 and with potential future development. We discuss imperfect background calibration due to temporal and spatial variability and the sensitivity of exozodi levels in Sec. 5 and conclude with future prospects in Sec. 6.

\section{Starshade Performance Model}

Figure 1 provides an overview of the geometry of starshade-enabled exoplanet imaging and an overview of the background and noise sources. Regardless of the specifics of telescopes, the $S / N$ of exoplanet direct imaging with a starshade is

$$
S / N=\frac{N_{P}}{\sqrt{N_{P}+\alpha\left(N_{S} C+N_{G}+N_{E}+N_{Z}+N_{D}\right)+\beta^{2}\left(N_{S} C+N_{G}+N_{E}+N_{Z}+N_{D}\right)^{2}}},
$$

where $N_{P}, N_{S}, N_{G}, N_{E}, N_{Z}$, and $N_{D}$ are the counts from the planet, the star, the solar glint, the exozodiacal dust, the local zodiacal dust, and detector noise, respectively, and $C$ is the instrument contrast. The parameters $\alpha$ and $\beta$ in Eq. (1) result from background subtraction, which is discussed in Sec. 2.1. The stray light sources shown in Fig. 1 but not included in Eq. (1) are discussed in Sec. 3. The counts are defined by a photometric aperture, which in turn corresponds to the point spread function (PSF) of the telescope. We assume a photometric aperture diameter of $\lambda / D$, which is consistent with the S5 milestone reports. ${ }^{9-11}$ Our choice of photometric aperture

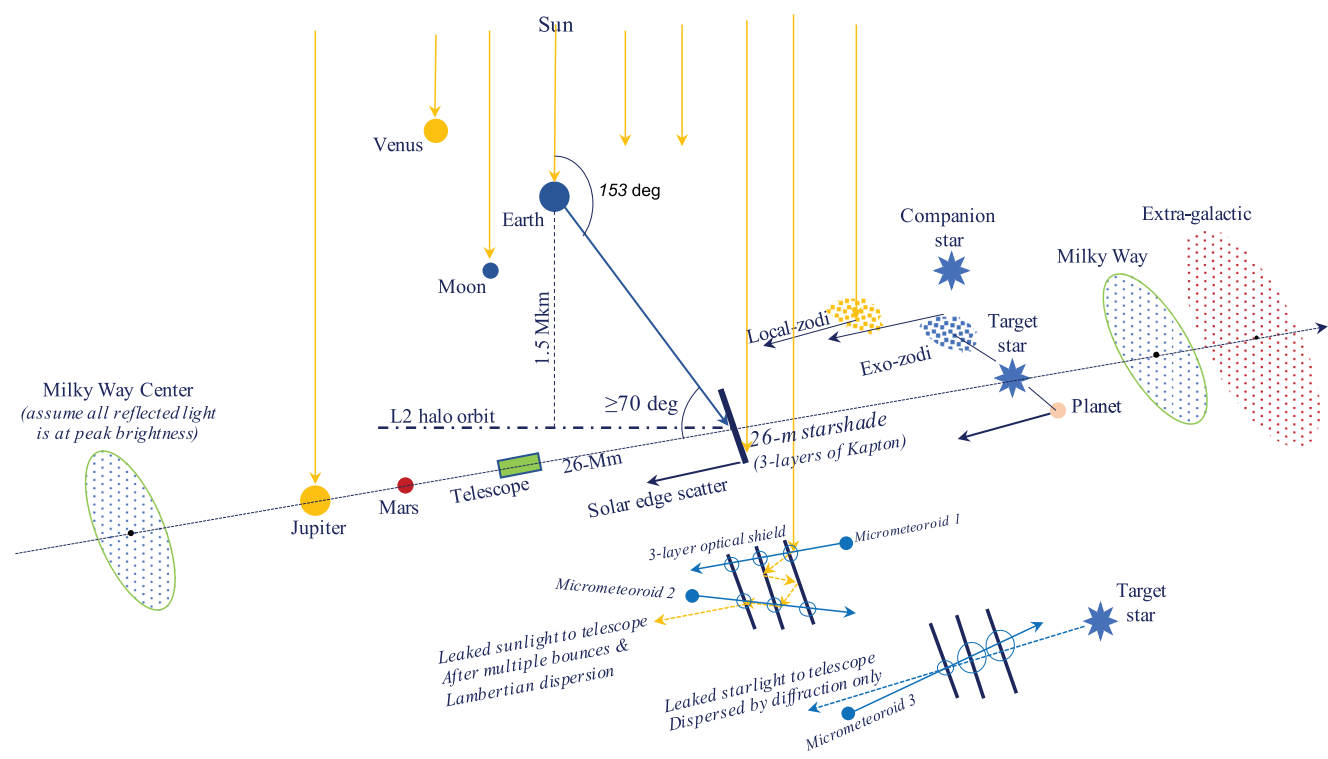

Fig. 1 The geometry of starshade-enabled exoplanet imaging and overview of stray light sources. Sunlight scattered to the telescope by the starshade's optical edge (i.e., the solar glint) is the dominant stray light source, followed by the reflection of the Milky Way, Earth, and other bright bodies in the solar system. Micrometeoroids can produce holes on the starshade's OS and cause leakage of sunlight and starlight. In exoplanetary systems, the exozodiacal dust can scatter the host star's light to the telescope. Finally, other more distant stars and galaxies may appear on the image and cause confusion. The quantities specified are for Starshade Rendezvous with Roman, but those of HabEx are qualitatively similar. We provide a comprehensive analysis of these light sources and their impact on the science performance in Secs. 2-4. 
Table 1 Parameters adopted in this work.

\begin{tabular}{|c|c|c|}
\hline Parameter & Roman & HabEx \\
\hline \multicolumn{3}{|l|}{ Starshade } \\
\hline Diameter (m) & 26 & 52 \\
\hline Distance to telescope (Mm) & 25.7 & 76.6 \\
\hline IWA (mas) & 104 & 70 \\
\hline Contrast at IWA & $10^{-10}$ & $10^{-10}$ \\
\hline Solar glint magnitude ${ }^{a}$ at IWA & $24.6(615$ to $800 \mathrm{~nm})$ & $27.2(300$ to $1000 \mathrm{~nm})$ \\
\hline Solar glint magnitude ${ }^{a}$ with coating & 27.1 & 29.7 \\
\hline \multicolumn{3}{|l|}{ Telescope } \\
\hline Aperture (m) & 2.4 & 4.0 \\
\hline End-to-end throughput & 0.03 & 0.2 \\
\hline Detector dark current (e/pix/s) & $3 \times 10^{-5}$ & $3 \times 10^{-5}$ \\
\hline Detector clock-induced charge (e/pix/frame) & $1.3 \times 10^{-3}$ & $1.3 \times 10^{-3}$ \\
\hline Pixels per spectral element & 42 & 56 \\
\hline \multicolumn{3}{|l|}{ Observation } \\
\hline Wavelength $(\mathrm{nm})$ & 700 & 700 \\
\hline Spectral resolution & 70 & 140 \\
\hline \multicolumn{3}{|l|}{ Planet } \\
\hline Geometric albedo & 0.3 & 0.3 \\
\hline Phase angle (deg) & 60 & 60 \\
\hline Phase function & Lambertian & Lambertian \\
\hline Exozodi dust level & 3 zodis & 3 zodis \\
\hline
\end{tabular}

aThe magnitude is defined such that a 25 -magnitude glint has the same energy flux in the wavelength band in parenthesis as a solar-spectrum point source that has a magnitude of 25 relative to an AOV star, analog to the Johnson magnitude system.

would encircle $46 \%$ of the flux from a point source and is smaller than Refs. 15 and 17, which is $1.4 \lambda / D$. The $S / N$ yielded from the $\lambda / D$ photometric diameter is $\sim 7 \%$ less than the theoretical maximum achieved at $1.4 \lambda / D$ in the background-limited regime. The aperture size in reality may eventually be controlled by the fixed pixel scale of the detector. Parameters used to estimate the contribution of the background sources and the expected $S / N$ are summarized in Table 1.

\subsection{Background Removal}

The quality of background subtraction is characterized by the parameters $\alpha$ and $\beta$ in Eq. (1). When $\beta=0$, the background can be subtracted from the photon-noise limit, where $\alpha$ describes the fractional increase from the photon noise that would manifest in the result. For example, if image processing can be approximated by subtracting two adjacent pixels of equal background, one with the planet and the other without, $\alpha=2$. For another example, if many more pixels can be used to characterize the background, $\alpha$ would approach unity. One may thus reasonably expect that, with largely smooth and static background, $\beta \rightarrow 0$ and $\alpha \sim 1-2$. Note that all counts in Eq. (1) are proportional to the integration time $(\Delta T)$. When $\beta \rightarrow 0, S / N \propto \Delta T^{-1 / 2}$. 
Imperfect background removal leads to a small but non-zero $\beta$. Let $K$ be $N_{P} / N_{B}$, where $N_{P}$ is the count from the planet and $N_{B}$ is the count from the dominant background or noise term. Equation (1) can be converted to the following form:

$$
S / N=\frac{S / N_{0}}{\sqrt{1+\frac{\beta^{2}}{K^{2}}\left(S / N_{0}\right)^{2}}},
$$

where $S / N_{0}$ is the $S / N$ when $\beta=0$. Equation (2) expresses how much $S / N$ would degrade due to imperfect background calibration. Several insights can be observed from Eq. (2). First, when $K \gg 1$ (i.e., the planet dominates over the background), the $S / N$ is not prone to degradation due to imperfect background calibration. Second, when $K \ll 1$ (i.e., the background dominates over the planet), or at large $S / N_{0}$ (i.e., large $\Delta T$ ), the asymptotic $S / N$ would be $S / N \rightarrow K / \beta=N_{\mathrm{P}} / \beta N_{\mathrm{B}}$. Third, at the critical case of $\beta=\frac{K}{S / N_{0}}$, the $S / N$ would become $S / N_{0} / \sqrt{2}$, i.e., degraded by a factor of $\sqrt{2}$. Note that the value of $\beta$ can be different for each source of background; as we show later, one source usually dominates for each observation. Here we neglect potential interference between the noise sources and its potential contribution to the $\beta$ term.

The analysis here tells us that the fundamental limit of planet detection is determined by the flux ratio between the planet and the dominant background source [ $K$ in Eq. (2)], as well as how well image processing can subtract the background from the photon-noise limit $[\beta$ in Eq. (2)]. Causes for not achieving the photon-noise limit include detector systematics (as is the case for transit observations with Hubble and Spitzer ${ }^{18}$ ) and temporal variability of speckles in coronagraphic direct imaging. ${ }^{19}$ These causes do not apply to future starshade direct imaging because of the use of EMCCD and the decoupling between starlight suppression from telescope optics. The capability of deep imaging provided by starshade may however render other causes to be the limiting factor, for example, the temporal variability of residual starlight and solar glint and the spatial distribution of exozodiacal dust. We adopt $\alpha=2$ and $\beta=0$ in the analyses that follow and discuss this point in Sec. 5 .

\subsection{Residual Starlight}

Based on the S5 milestone reports, ${ }^{9,10}$ we adopt a contrast ratio of $10^{-10}$ at the IWA resulting from an imperfect starshade. As the HZs of nearby stars are often substantially larger than the IWA, we use the Starshade Imaging Simulation Toolkit for Exoplanet Reconnaissance (SISTER $^{20,21}$ ) to determine the instrument contrast as a function of the angular separation (Fig. 2) and use the results in the subsequent analyses. The residual starlight drops with the angular separation as $\sim \theta^{-3.4}$, where $\theta$ is the off-axis angle. This is slightly steeper than the expected Airy pattern drop of $\theta^{-3}$ for a filled aperture due to the distribution of the residual starlight (i.e., some near the center of the starshade and some localized near the petals). Finally, an off-axis companion beyond the IWA does not experience a change in transmission for starshade observations. ${ }^{20}$

\subsection{Solar Glint}

Although the Sun will not be on the telescope-facing side of the starshade, the starshade's edges will scatter sunlight toward the telescope via a combination of diffraction, diffuse reflection, and specular reflection. ${ }^{23}$ When these scattering mechanisms are considered together, the telescope sees the scattered sunlight coming mainly from localized regions on a few petals where the optical edge is aligned for specular reflection (Fig. 1). These appear as two broad lobes due to the telescope's finite spatial resolution, i.e., the solar glint. The solar glint is a unique effect in direct imaging using a starshade.

We adopt the brightness of the solar glint measured by S5 with a razor-sharp, amorphous metal edge ${ }^{11}$ and use SISTER to calculate its angular dependency (Fig. 2). The expected magnitudes of the solar glint are calculated by combining scattering measurements of prototype optical edge segments and the optical models of the starshade. ${ }^{11}$ We adopt a "worst case" scenario, 


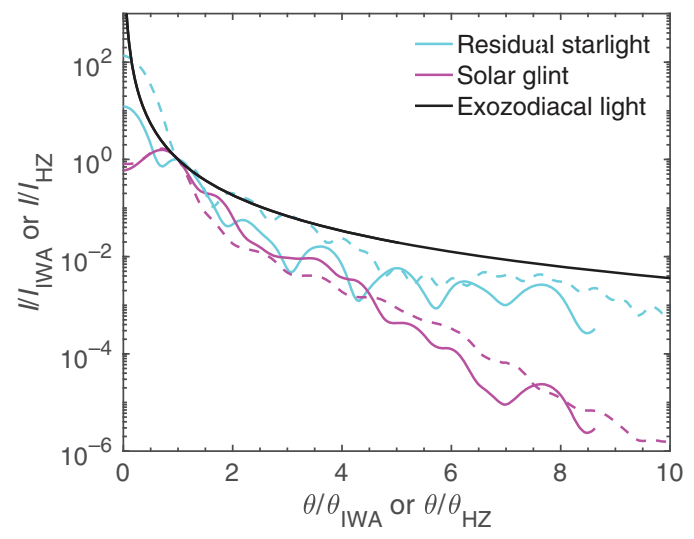

Fig. 2 Dependencies of residual starlight, solar glint, and exozodiacal light on angular separation. The solid lines are for a 26-m starshade coupled with Roman and the dashed lines are for a 52-m starshade of HabEx. The residual starlight and solar glint shown are azimuthal averages and scaled to the IWA, and their dependencies on the off-axis angle $\theta$ are calculated using SISTER. For Roman, the IWA in the green band (615 to $800 \mathrm{~nm}$ ) is 104 mas, corresponding to a 13-m radius at a distance of $26 \mathrm{Mm}$. For Habex, the IWA is 70 mas. This scaling conveniently lets one describe the residual starlight and solar glint, the source of which is always located within the geometrical starshade pattern, in terms of apparent starshade radius. The exozodiacal light is scaled to the $\mathrm{HZ}$, and its angular dependency is calculated with Zodipic ${ }^{22}$ assuming a solar-system dust density profile.

i.e., the maximum solar angle ( $\sim 83 \mathrm{deg})$ and $95 \%$ confidence upper limit of the brightness at the IWA. ${ }^{11}$ The expected brightness of the solar glint for HabEx is approximately one-order-ofmagnitude less than that for Roman. This is because the starshade of HabEx is much more separated from the telescope than the starshade from Roman (Table 1). The solar glint features a hump that peaks at the IWA and quickly drops as the angular separation increases and thus impacts planet search near the IWA most significantly.

Recognizing the potential impact of the solar glint on science performance, S5 and its Science and Industry Partnership have been actively seeking improvement on the optical edge technology. A multi-layer, thin-film coating has recently emerged as a highly promising design, and experiments have indicated that the coating results in a solar glint brightness lower than the uncoated design by approximately one order of magnitude. ${ }^{24}$ We also study the science performance with the solar glint brightness suppressed by the coating.

\subsection{Exozodiacal Light}

Results from the most sensitive exozodiacal dust survey indicate that the majority of nearby Sunlike stars have relatively low HZ dust levels, with the best-fit median being 3 times the solarsystem level, whereas some stars (e.g., $\epsilon$ Eridani) are significantly more dusty. ${ }^{25}$ In this work, we assume " 1 zodi" corresponds to $22 \mathrm{mag}$ arc sec in the $\mathrm{V}$ band at the $\mathrm{HZ}^{17}$ and typically assume " 3 zodis" in the analysis. This assumption was also adopted by mission concept studies and exoplanet yield analyses. ${ }^{6,715}$ We discuss the sensitivity of the exozodi levels in Sec. 5.3. We also assume that the brightness of the exozodiacal light is independent of the distance or the stellar type if evaluated at the $\mathrm{HZ}$ (unless otherwise noted, the $\mathrm{HZ}$ in this paper refers to the orbital distance that yields the same stellar flux as $1 \mathrm{AU}$ from the Sun, i.e., the 1-AU equivalent), besides a factor from the spectral shape of the star. ${ }^{17}$ Scaling to the HZ, we approximate the brightness of the exozodiacal light as dependent on the semimajor axis at $a^{-2.44}$. This scaling is based on example "Zodipic" simulations with a solar-system dust density profile. ${ }^{22}$

\subsection{Detector Noise}

We model the detector noise as the combination of dark current and clock-induced charge. ${ }^{15}$ The EMCCD would have effectively zero read noise. We assume the frame rate to be 6.73 times the 
count rate of the brightest pixel. ${ }^{15}$ This is to ensure that the probability of two photons arriving at the brightest pixel is $<1 \%$, at the expense of increasing the clock-induced charge. The number of pixels for each spectral element is assumed to be 42 for Roman and 56 for HabEx. These are estimated for spectral characterization at $700 \mathrm{~nm}$ with the assumptions of a PSF core of 4 pixels, dispersed into 6 pixels per spectral elements, and the detector providing Nyquist sampling at $400 \mathrm{~nm}$ for Roman and $300 \mathrm{~nm}$ for HabEx. ${ }^{15}$

\section{Overview of Stray Light}

Here we overview other noise terms not accounted for in the primary noise budget [Eq. (1)].

\subsection{Astrophysical Background}

The very dark shadow created by the starshade reveals not only planets but also faint stars and distant galaxies. The Exo-S final repor ${ }^{26}$ provided a detailed analysis of background star and galaxy confusion and suggested mitigation strategies. If planets within $5 \mathrm{AU}$ from the parent stars are potentially detectable (farther planets would often be too faint in reflected light), the largest area for planet search would be $\sim 6$ arc sec. One can expect $\sim 2$ distant galaxies down to $V \sim 31^{27}$ and $\sim 0.2$ stars in this area depending on the galactic latitude. ${ }^{26}$ Stars with known companions that have small angular separation and would impact direct imaging have been excluded from the target lists of Starshade Rendezvous ${ }^{6}$ with Roman and HabEx. ${ }^{7}$ Spectra may provide clues to tell the planets apart from these background sources, and revisits and the detection of common proper motions with the parent star would be required to confirm the planets.

\subsection{Micrometeoroid Holes}

Micrometeoroids can penetrate the starshade's multi-layer opaque optical shield (OS). The baseline OS design consists of three evenly spaced layers of Black Kapton. Some large or highvelocity micrometeoroids can produce through-holes on the starshade. During a science integration, on-axis starlight can pass directly through the fraction of particle trajectories aligned to the starshade normal to disperse only via diffraction toward the telescope (Fig. 1). Off-axis sunlight instead requires multiple reflections within the OS before exiting to disperse in the Lambertian fashion toward the telescope (Fig. 1). It is thus necessary to consider micrometeoroid holes and the transmission of starlight and sunlight through them.

We developed a model to estimate micrometeoroid holes and the resulting brightness levels for Starshade Rendezvous with Roman. The mass-flux distribution of micrometeoroids at L2 is estimated with the Grun model. ${ }^{28}$ The Grun model does not account for seasonal meteor showers that bring elevated fluxes by 1 to 2 orders of magnitude. It may be necessary to orient the starshade to a near edge-on direction during one or two showers per year, for a total of approximately 1 month per year. The lost observation time can be mitigated to some extent by the timing of retargeting maneuvers with long coast periods.

The minimum particle mass required to enter the OS is computed with a single-layer ballistic equation from NASA's Preferred Reliability Practices document for micrometeorite protection (Standard PD-EC-1107). The minimum particle mass to pass the middle layer and then exit the third layer is computed with a two-layer ballistic equation. ${ }^{29}$ The exit computation conservatively neglects the benefit of the middle layer. The incoming flux is assumed to be isotropic in direction considering variable starshade pointing throughout the mission. The particle specific density is conservatively bounded by a constant of $2.5 \mathrm{~g} \mathrm{~cm}^{-3}$ for considering the ability to penetrate the OS and $1 \mathrm{~g} \mathrm{~cm}^{-3}$ for considering the size of holes produced. Entry hole diameters approximately match the particle diameter for large particles and can be greater than the particle diameter by up to a factor of five for small particles. ${ }^{30}$ When a high-velocity particle penetrates the first layer, the particle and the shield material vaporize, creating an expanding gas cloud. A subset of the gas cloud can then penetrate the middle and exit layers. We estimate that the exit and middle-layer hole diameters can grow by up to a factor of thirty from the original particle diameter to account for the gas cloud expansion. ${ }^{31}$ 
Our model indicates that the hole areas after three years on-orbit would be 0.1 parts-permillion (ppm) by surface area for entry holes on both sides, $50 \mathrm{ppm}$ for exit holes on both sides, and $150 \mathrm{ppm}$ for middle-layer holes. The expected number of entry holes is about $5 \times 10^{5}$, whereas the expected number of exit holes is $<400$. The exit holes are produced only by large particles.

We estimate that $\sim 10 \%$ through-holes would provide a direct path for starlight to the telescope. The leakage is limited by the entry hole area on both sides yielding $0.02 \mathrm{ppm}$ of effective area. Feeding this area to our optical performance model of starshades,$^{32}$ we estimate that the starlight leakage due to micrometeoroid holes corresponds to a residual starlight contrast of $10^{-13}$, lower than the allowed residual starlight contrast by three orders of magnitude.

We then estimate the upper bound of solar transmission to be the product of the porosity factors of the layers and obtain $4 \times 10^{-13}$. The solar leakage is also proportional to the micrometeoroid hole area but not necessarily to the number of holes. This transmission corresponds to a brightness of solar leakage at the telescope that has a visual magnitude of 39.6 at the IWA at $700 \mathrm{~nm}$, with the brightness falling off with the off-axis angle at approximately the same rate as for the solar glint. We thus expect the solar leakage due to micrometeoroid holes to be dimmer than the solar glint by $>10$ magnitudes.

Although the estimates apply for Starshade Rendezvous with Roman, they can be scaled for HabEx considering a longer (5 years) mission lifetime and a higher angular resolution (defined by $\lambda / D$ ). The distance from the starshade to the telescope does not appear in this scaling because the solid angle per resolution element is independent of the distance. We estimate that the brightness from micrometeoroid holes on HabEx's starshade would have approximately the same magnitude as the Starshade Rendezvous and would remain much dimmer than the solar glint.

\subsection{Bright-Body Reflections}

Bright celestial bodies positioned on the telescope side of the starshade may have a portion of their light reflected toward the telescope, with the brightness falling off with the off-axis angle at approximately the same rate as for solar glint. The starshade presents mostly Black Kapton to the telescope, and we evaluate bright-body reflections with its BRDF data. To estimate the magnitude of bright-body reflections, we start from a solar-system planet's absolute magnitude and consider the worst case orbit phasing to derive the brightness incident at the starshade. We then take into account the angular size of the starshade and its reflectivity in the direction of the telescope. Finally, we distribute the brightness into each resolution element on the starshade as the starshade would have nearly uniform brightness in this problem. Table 2 provides a detailed list of our estimates of bright-body reflections for solar-system bodies.

Light from Earth, Moon, and Venus can be reflected toward the telescope at grazing incidence angles, but only at the extremes of starshade's orbit position and pointing (Fig. 1). Earth and Venus maximally can appear at about the same magnitudes of 31.7 mags at the IWA (Table 2). To have this magnitude, Venus must be at the quadrature orbital phase viewed from the starshade when the starshade is at one extreme of its L2 orbit, a combination that rarely occurs. The Moon will be much dimmer than Earth and Venus for starshade reflection to the telescope.

Light from Mars and Jupiter can be reflected toward the telescope at nearly normal incidence angles (Fig. 1). We find that Jupiter never exceeds a brightness of 31.9 mags at the IWA (Table 2), and Mars is much dimmer.

In addition to the solar-system bodies, the integrated light from the center of the Milky Way is likely the brightest object that can be reflected toward the telescope by the starshade. The Milky Way can appear as bright as 20.6 visual magnitudes per $\operatorname{arc~sec}^{-2},{ }^{34}$ or 27.0 mags per resolution element at $700 \mathrm{~nm}$. The starshade's hemispherical reflectance is about $5 \%$. Assuming that all of the reflected light comes from the brightest part of the Milky Way and adjusting for the starshade not being a full disk, we estimate the maximum brightness to be 31.3 mags at the IWA.

In all, we find that the bright-body reflections combined are no brighter than a magnitude of $\sim 30$ at the IWA as a conservative estimate for Starshade Rendezvous with Roman. The bright-body reflections for HabEx should be dimmer by a factor of $\sim 2$. The bright-body reflections thus constitute a stray light source dimmer than the solar glint (with coating 
Table 2 Estimates of Earth, Venus, and Jupiter brightness reflected by the starshade to the telescope at their orbital positions and the starshade's position and pointing that maximize these stray light sources. The estimates use the parameters of Starshade Rendezvous with Roman, and the result for HabEx scales by the solid angle per resolution element.

\begin{tabular}{lcccc}
\hline \hline Parameter & Units & Earth & Venus & Jupiter \\
\hline Absolute magnitude $(H)^{\mathrm{a}}$ & mags & -3.99 & -4.38 & -9.40 \\
Sun distance & $\mathrm{AU}$ & 1 & 0.72 & 5.2 \\
Starshade (SS) distance & $\mathrm{AU}$ & 0.0112 & 0.70 & 4.55 \\
Maximum phase angle at SS & deg & 153 & 90 & 9.3 \\
Phase function in SS direction & mmags & 4.65 & 1.43 & 0.05 \\
Brightness incident at SS & mags & -9.10 & -4.51 & -2.57 \\
Incidence angle from SS normal & deg & 70 & 50 & 0 \\
SS reflectivity in telescope direction & per s.r. & 0.00261 & 0.1 & 0.3 \\
SS area/distance & (2) & 30.8 & 30.8 & 30.8 \\
Total brightness at telescope & mags & 29.2 & 29.1 & 29.4 \\
\# resolution elements on SS & & 10 & 10 & 10 \\
Brightness per resolution element & mags & 31.7 & 31.6 & 31.9 \\
\hline \hline
\end{tabular}

The absolute magnitude of a solar-system object is defined as the apparent magnitude that the object would have if it were $1 \mathrm{AU}$ from both the Sun and the observer and in conditions of ideal solar opposition. ${ }^{33}$

improvement) by approximately one order of magnitude for Starshade Rendezvous and a factor of 2 for HabEx.

\subsection{Other Stray Light}

Other stray light may include secondary solar reflections, fluorescence, thruster exhaust solar scatter, and stray light produced by the telescope. The first three potential sources are subjects of starshade technology, and our preliminary analyses indicate that these light sources likely contribute to the background by no more than a fraction of the solar glint. S5 is conducting extensive stray light analyses to evaluate possible stray light paths and, if necessary, adjust the starshade design and the observation constraints to mitigate their impacts.

The current starshade design is intended to preclude any sunlight reaching the telescope after only a single reflection, except for the optical edges (i.e., the solar glint). However, secondary solar reflections, where sunlight can reach the telescope after two reflections, are possible with certain particular light paths, and they are being studied by S5. Preliminary analysis suggests an acceptable brightness level. For example, some stray light paths involve an out-of-plane deformed petal, and stray light analyses are being developed to establish a tighter, yet still readily achievable, requirement of how far the petal can deform out-of-plane versus the current specification based on diffraction performance alone. For another example, some solar leakage may occur through small gaps between the OS at the inner disk to the petal interface. This interface is dynamic at the end of disk deployment as a hard tie-down point rotates into node plates at the ends of each inner disk facet. A labyrinth seal is designed to attenuate the sunlight with a preliminary verification of performance, and a more thorough stray light analysis is planned to confirm this design.

Black Kapton used in the starshade's OS is known to glow in response to high-energy solar electrons due to material fluorescence. Informed by past studies for JWST, we estimate a relatively dim magnitude of 33.3 at the IWA, i.e., dimmer than the solar glint (with coating) by two 
orders of magnitude. Efforts are underway to better understand the input electron energy levels and evaluate the fluorescence of Black Kapton and other material refinements.

Periodic thruster firings to maintain formation flight are expected about once every $10 \mathrm{~min} .{ }^{12}$ Exhaust particles scatter sunlight at a level that can saturate detectors. An operating concept to prevent detector saturation is for the starshade to notify the telescope of an imminent thruster firing and for the telescope to switch to a fast detector readout mode for some conservative duration (e.g., $10 \mathrm{~s}$ ). This is more than enough time for the bulk of the exhaust to leave the field of view and only causes a modest loss of observing time. Finally, large exhaust particles might "loiter" around the starshade or stick to the exhaust nozzle to be pushed off at relatively low velocity at the next thruster firing. The optical density of these loitering particles would be much lower than the high-velocity exhaust particles, and detailed simulations are required to assess their brightness.

\section{Results and Analyses}

We choose stars that represent the target lists of Starshade Rendezvous ${ }^{6}$ and HabEx ${ }^{7}$ in this study. As seen in Fig. 3, the nearest stars for the search of potentially habitable planets may be grouped into three distance groups: $<4$ parsecs, 5 to 6 parsecs, and $\sim 8$ parsecs. Within each group, Starshade Rendezvous with Roman would search for habitable-zone planets around stars from A/F to late G/early K spectral types. HabEx would extend the search to stars of late $\mathrm{K}$ and even early $\mathrm{M}$ spectral types, in addition to many more distant stars. Most nearby stars are $\mathrm{M}$ type stars, and they are generally not amenable for direct imaging of planets in the HZ due to IWA restrictions. The example stars used in this study cover the distance groups and the spectral type (and luminosity) ranges of the target stars of Starshade Rendezvous with Roman and HabEx.

For each representative star, we evaluate the detectability of planets of varied size and planetstar separation. Rather than focusing on $1-R_{\oplus}$ planets that receive Earth-like insolation, we include larger planets at colder or hotter orbits as potential search targets. This is motivated by the diversity of planets revealed by current exoplanet searches and improved understanding of the HZs. Kepler planet surveys and planetary mass measurements available for a subset of the detected planets have indicated two populations of small planets. ${ }^{35}$ The planets in the $<1.7 R_{\oplus}$ population are mostly rocky, ${ }^{36}$ and the planets in the 1.7 to $3.0 R_{\oplus}$ population are planets that could have $\mathrm{H}_{2} / \mathrm{He}$ gas envelopes ${ }^{37,38}$ and/or substantial water layers. ${ }^{39,40}$ The larger planets, if they have moderate-size atmospheres, can host liquid-water oceans and thus be potentially habitable. ${ }^{41,42}$ The "HZs" for planets with $\mathrm{H}_{2}$-dominated atmospheres can be substantially more

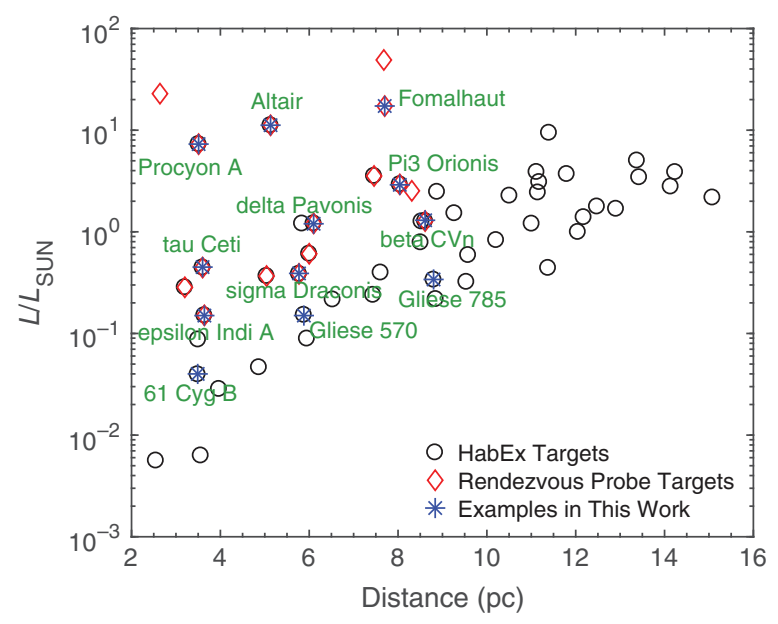

Fig. 3 Nearby stars for the search of potentially habitable planets adopted by Starshade Rendezvous ${ }^{6}$ and HabEx. ${ }^{7}$ Example stars used in this study are labeled. Although many more $M$ type stars can be found in the distance range shown, their HZs have smaller angular separations than the IWA of Starshade Rendezvous with Roman or HabEx. 
separated from the parent stars than those for planets with $\mathrm{N}_{2^{-}}$and $\mathrm{CO}_{2}$-dominated atmospheres. ${ }^{41,43}$

We focus on the spectral characterization of exoplanets in this paper. We adopt a requirement of $S / N=20$ per spectral element as this is a conservative estimate of the precision needed to measure atmospheric abundances from the reflected-light spectra and potentially distinguish the types of planets. ${ }^{44-49}$ The expected integration time to detect planets in the broadband is substantially less than what is shown in this section. Also all results shown in this section assume unbiased calibration of the background to the photon-noise limit. We discuss background calibration in Sec. 5 .

\subsection{Current Performance of Starshade Rendezvous}

Figure 4 shows the expected integration time and the underlying dominant noise source to detect planets around nearby stars with demonstrated starshade optical performance and telescope parameters that approximately correspond to the Starshade Rendezvous with the Roman mission concept (see Table 1). We make the following observations from Fig. 4. First, exozodiacal light is the dominant noise term for planet searches around nearby F and A stars. These early-type stars have widely separated HZs, and thus the habitable-zone planets have small planet-to-star contrast. Also because we assume the exozodiacal brightness scales to the HZ (the 1-AU equivalent in this context), the expected exozodiacal light brightness near the IWA is high for these earlytype stars.

Second, for the search of small $\left(<2.5 R_{\oplus}\right)$ planets near the HZs of the nearest late $\mathrm{G}$ and $\mathrm{K}$ stars (i.e., $\tau$ ceti and $\epsilon$ indi A), the planet itself is likely the dominant noise term. In other words, the current performance expected for Starshade Rendezvous with Roman could provide a photon-limited detection, rather than background-limited detection, for temperate and small planets
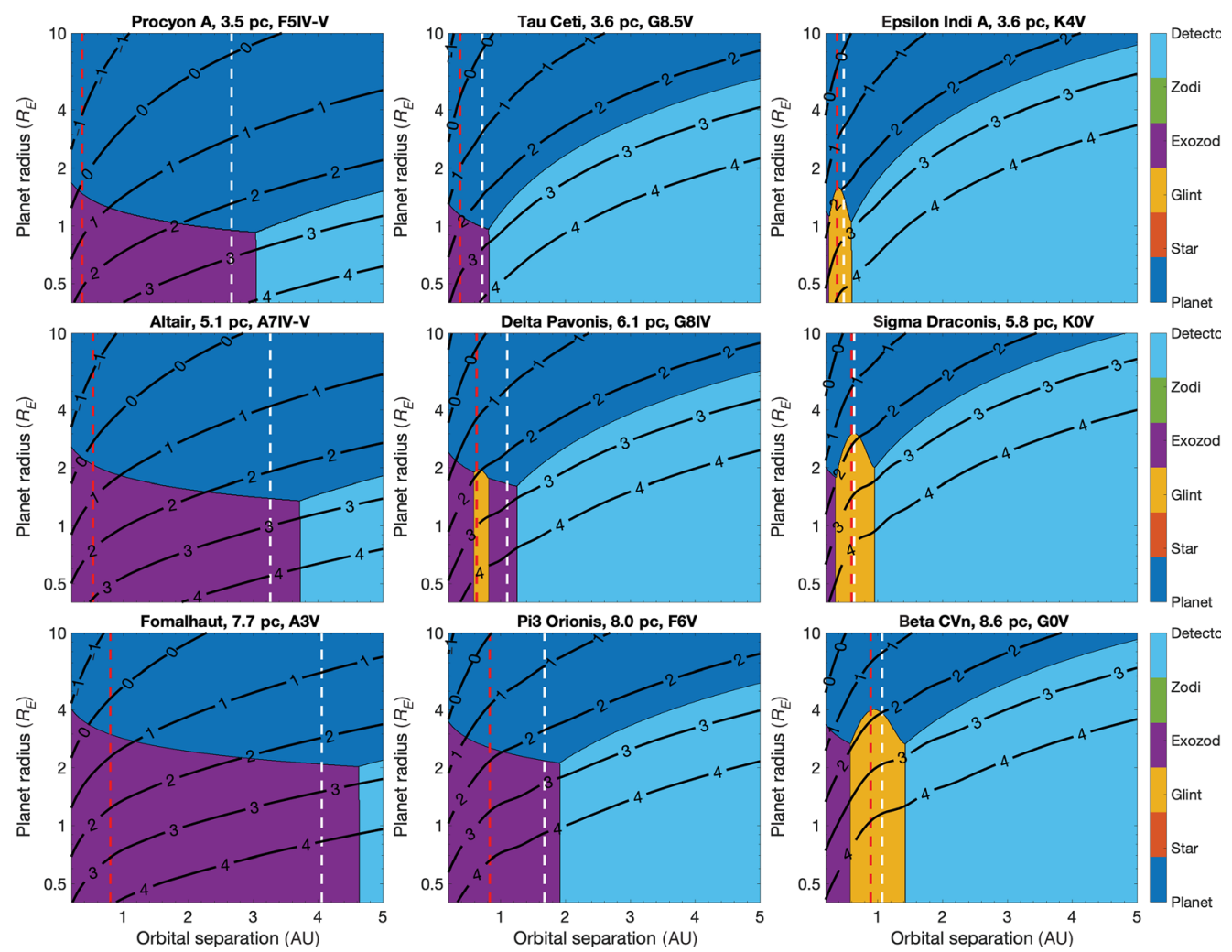

Fig. 4 Expected integration time $[S / N=20$ per spectral element, contour profiles, in base-10 log (hours)] and dominant noise source (shaded areas) expected in Roman and Starshade Rendezvous exploration of planets around nearby stars. The red dashed line corresponds to the IWA, and the blue dashed line corresponds to the planet-star separation for receiving Earth's insolation, i.e., the 1-AU equivalent. Parameters of the simulations are shown in Table 1. 
around these most favorable targets. This is because the stars are less luminous than the Sun, which makes the HZs closer to the stars ( 0.4 to $0.7 \mathrm{AU})$. The planet-star contrast at their HZs is 7 to $20 \times 10^{-10}$ for $1-R_{\oplus}$ planets. Coupled with the brightness of these stars (visual magnitude of 3.5 to 4.7 ), the brightness of $1-R_{\oplus}$ planets in their HZs has a visual magnitude of $\sim 26.3$, i.e., much brighter than a "mean" scenario of 30 . As such, the common belief that the detection would be mainly limited by exozodiacal light does not apply for these most favorable targets. They are also the prime targets from an integration time standpoint: to spectroscopically characterize (with $S / N=20$ ) a $1-R_{\oplus}$ planet near the $\mathrm{HZ}$ only requires integration of a few hundred hours, and the integration time reduces to $\sim 100 \mathrm{~h}$ for larger rocky planets with a $1.5-R_{\oplus}$ radius. Among these stars, $\epsilon$ Eridani has an especially bright dust disk that would probably prevent the search of small planets. ${ }^{25} \tau$ ceti have two super-Earth-mass planets $\left(M \sin i \sim 3.9 M_{\oplus}\right.$ ) near the $\mathrm{HZ}$ detected by radial-velocity measurements, ${ }^{50,51}$ and an outer dust disk ( $>6 \mathrm{AU}$ ) detected by far-infrared and radio observations. ${ }^{52-54}$ The constraints on the orbital elements and masses of these planets would aid future characterization.

Third, for slightly farther stars (5 to 6 parsecs, e.g., 40 Eridani, $\delta$ Pavonis, 82 Eridani, and $\sigma$ Draconis), the search for small planets near the HZs is limited by exozodiacal light and solar glint. For the later-type stars with HZs close to the IWA, the search for habitable-zone planets is particularly affected by solar glint. With the current performance ${ }^{11}$ and assuming calibration of the solar glint to the photon-noise limit, to characterize a $1-R_{\oplus}$ planet near the $\mathrm{HZ}$ requires integration of a few thousand hours, i.e., likely infeasible for a realistic space mission. Characterizing a 1.5 to $2.5-R_{\oplus}$ planet, however, only requires an integration time of 100 to $1000 \mathrm{~h}$. Large rocky planets and more volatile-rich planets could thus be studied with spectroscopy, and thus these "super-Earths" represent the near-term opportunity for the search of habitable worlds on these stars. ${ }^{55}$

Finally, for stars $\sim 8$ parsecs away, planet characterization near the HZs is limited to planets not smaller than $\sim 2 R_{\oplus}$, and the integration time required for smaller planets is much longer than $1000 \mathrm{~h}$. The solar glint continues to be the dominant noise term, except for the F and A stars.

\subsection{Impact of Future Development}

Guided by the analyses presented in Sec. 4.1, we now turn to the impact of the starshade performance parameters on the science returns. Because the diameter and the distance to the telescope determine the theoretical limits in starlight suppression and the IWA, ${ }^{56}$ we focus on the non-ideal effects. Specifically, random mechanical imperfections raise the starlight suppression level (expressed in the residual starlight contrast), and sources of stray light, dominated by the solar glint, contribute to the background.

As discussed in Sec. 2.3, one may reasonably expect the actual solar glint brightness to be approximately one order of magnitude lower than the nominal value due to coating technologies. ${ }^{24}$ Also we have used a "worst" scenario in the analysis presented in Fig. 4, in that the Sun was at the maximum angle from the line of sight $(83 \mathrm{deg})$ and the planet was close to the position where the solar glint was maximized. An "average" scenario where the solar angle is less $(\sim 53 \mathrm{deg})$ and the planet is away from the glint maximum results in a reduction in the solar glint brightness by a factor of $\sim 3 .{ }^{11}$

Figure 5 shows the impact on the science performance from a 10 -fold reduction on the solar glint brightness. We find that this improvement drastically enhances the prospect of the search for small planets around later-type nearby stars. The solar glint would no longer be the dominant noise term for the planet observations around these stars. The exozodiacal light becomes the dominant noise term in place. Consequently, a decrease in the required integration time by a factor 2 to 3 for planet search near the IWA would be expected (Fig. 6). This improvement is particularly substantial for exoplanet science because this would reduce the time needed to characterize $1-R_{\oplus}$ planets in the HZs of stars 5 to 6 parsecs away from a few thousand to $\sim 1000$ h (i.e., from infeasible to marginally feasible) and would reduce the time needed to characterize $1.5-R_{\oplus}$ planets (i.e., large rocky planets) from $\sim 1000 \mathrm{~h}$ to a few hundred hours (i.e., from marginally feasible to highly feasible). This improvement also extends the search for planets around 8 parsec, Sun-like stars from $2-R_{\oplus}$ planets to $1.5-R_{\oplus}$ planets, i.e., into the regime of rocky planets. Together, these impacts enlarge the stellar sample that are amenable for searching 

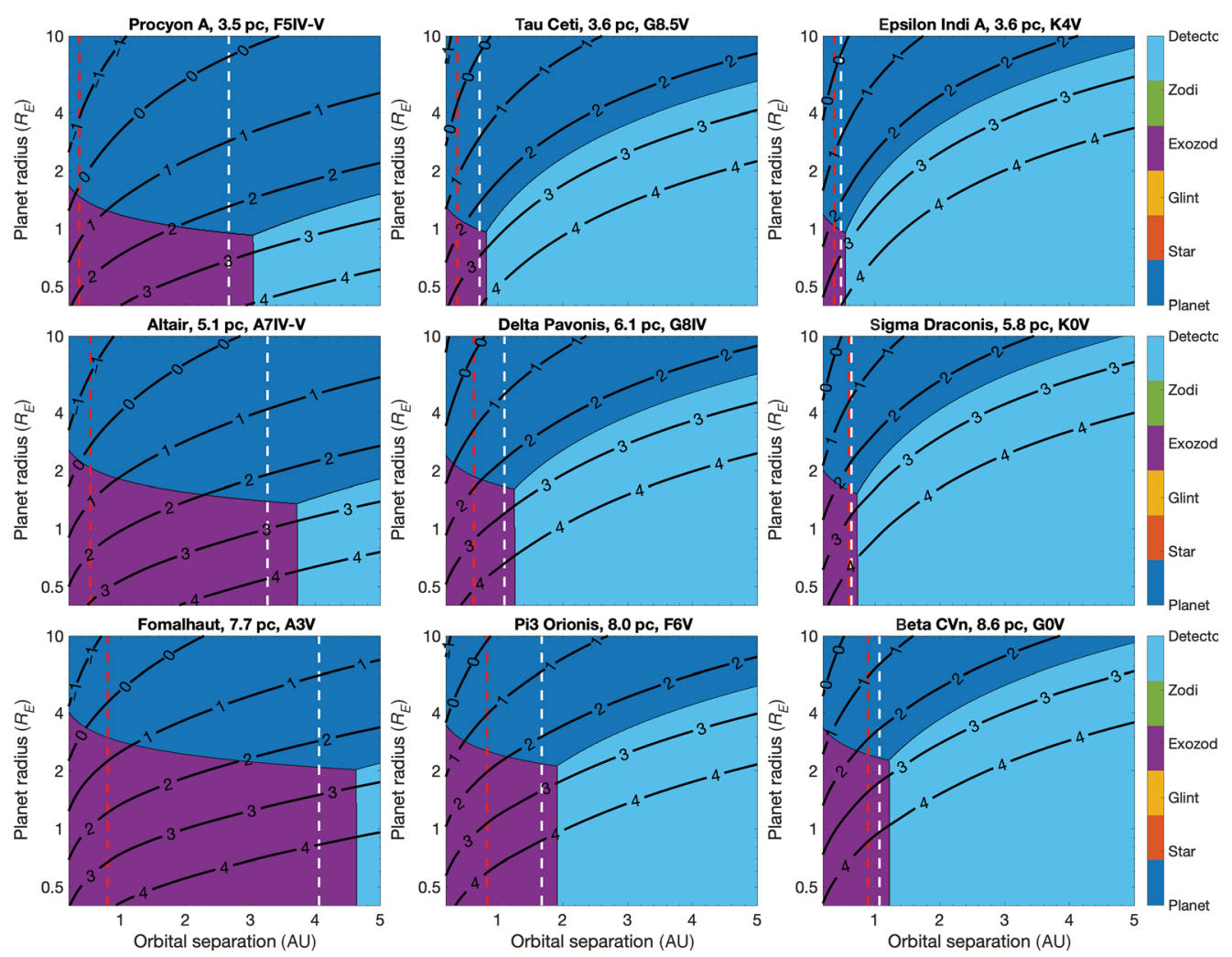

Fig. 5 The same as Fig. 4, except that the brightness of solar glint is lower than the nominal value by a factor of 10 .

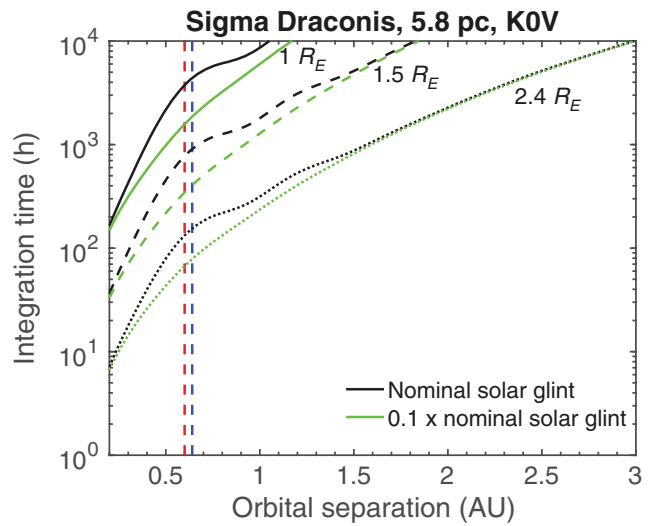

Fig. 6 Improvement in the required integration time to characterize $(S / N=20$ in a 10-nm wavelength channel) planets around a nearby star, from a 10-fold reduction of the solar glint brightness from the nominal value. This is essentially a zoom-in view of Fig. 5 for sigma Draconis. The red dashed line corresponds to the IWA, and the blue dashed line corresponds to the planet-star separation for receiving Earth's insolation, i.e., the 1-AU equivalent.

rocky planets in the HZs by a factor of at least three, thus substantially improving the science prospect.

For starlight suppression, the nominal performance shown in Fig. 4 does not have residual starlight as the dominant noise term anywhere in the explored parameter space. We are thus motivated to assess the tolerance of exoplanet characterization on a degraded residual starlight contrast. We have repeated the analysis with a contrast level that is 3,10, 30, and 100 times higher than the $10^{-10}$ level demonstrated by S5 experiments, on top of the low-solar-glint scenarios shown in Fig. 5. We find no appreciable change in the search of temperate and small 

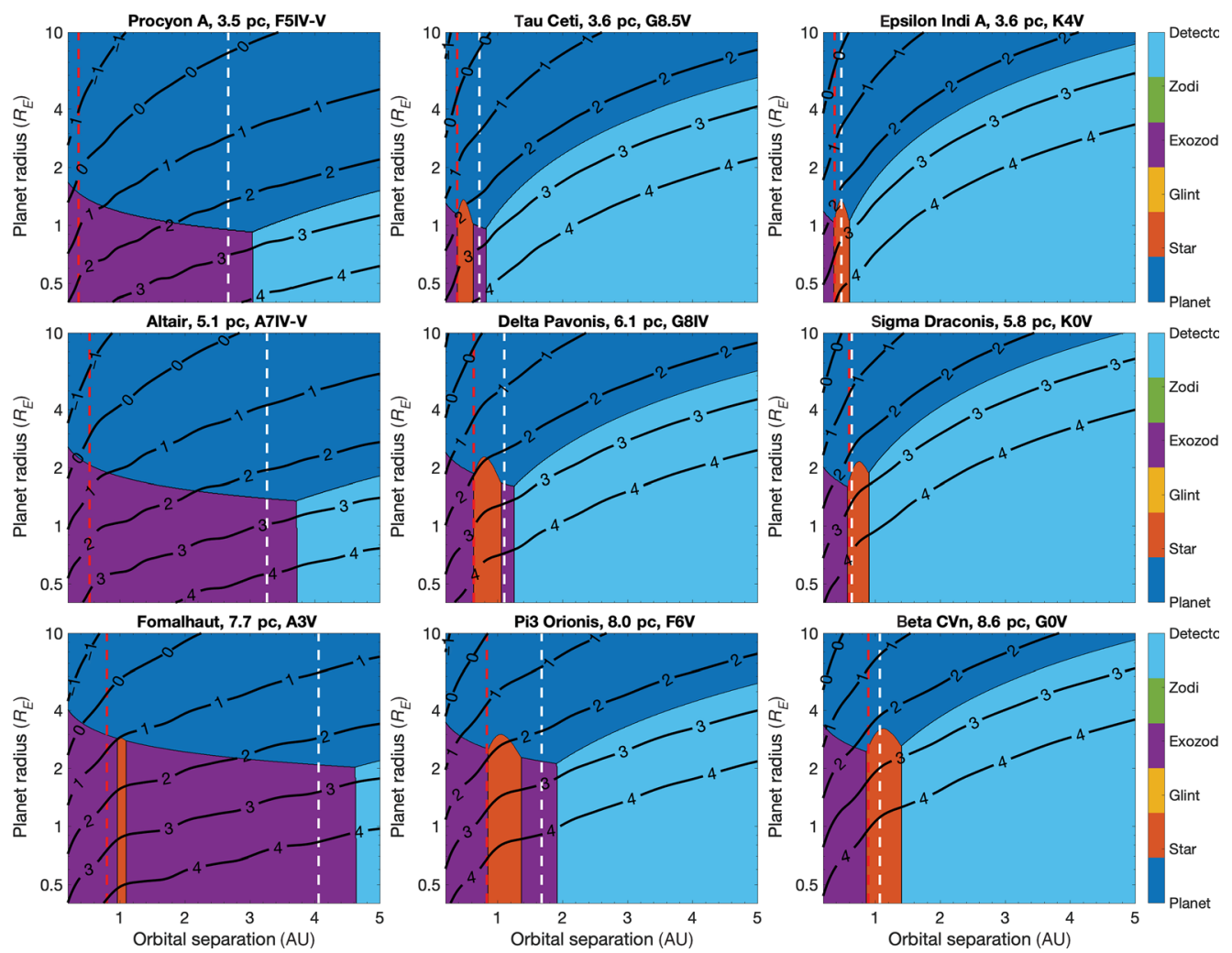

Fig. 7 The same as Fig. 4, except that the brightness of solar glint is lower than the nominal value by a factor of 10 and the residual starlight contrast is $3 \times 10^{-9}$ (i.e., higher than the nominal value by a factor of 30 ).

planets around nearby stars for up to 10 times worse contrast. Residual starlight would continue to be a non-dominating term in the noise budget, and the impact on the integration time would be minimal. Residual starlight would become the dominant noise term for observing 1 to $2 R_{\oplus}$ planets of nearby stars, associated with a moderate increase in the integration time to achieve an $S / N$ of 20 , when the contrast is $3 \times 10^{-9}$ (i.e., 30 times worse, Fig. 7). This analysis indicates that the $10^{-10}$ starlight contrast is sufficient, with one order of magnitude margin, for the science performance of the Starshade Rendezvous.

To summarize, the demonstrated starshade optical performance coupled with Roman would enable spectral characterization of small and temperate planets of nearby stars. With the coating technology to reduce the solar glint, the characterization of Earth-sized and Earth-temperature planets is possible for stars within 5 to 6 parsecs, and the characterization of temperate and large rocky planets of $1.5 R_{\oplus}$ is possible for stars as far as $\sim 8$ parsecs. A contrast level of $10^{-10}$ at the IWA is sufficient for these science applications as the residual starlight appears nowhere in the parameter space as the dominant noise term. For a uniform dust level of 3 zodis, the dominant noise term for the small planet characterization is likely exozodiacal light. The impact of exozodiacal light is larger for farther stars.

\subsection{Expected Performance of HabEx}

Figure 8 shows the expected integration time and the underlying dominant noise source to detect planets around nearby stars with demonstrated starshade optical performance and telescope parameters that approximately correspond to the HabEx mission concept (Table 1). Based on nominal performance parameters, solar glint would be the dominant noise term for observing Earth-sized and smaller planets in the HZs of the latest-type stars in each distance group. This is because the HZs of these stars approach the IWA, where the solar glint is the brightest. If the optical edge coating is applied and the solar glint brightness is reduced by a factor of 10 , this impact of the solar glint would be eliminated. 

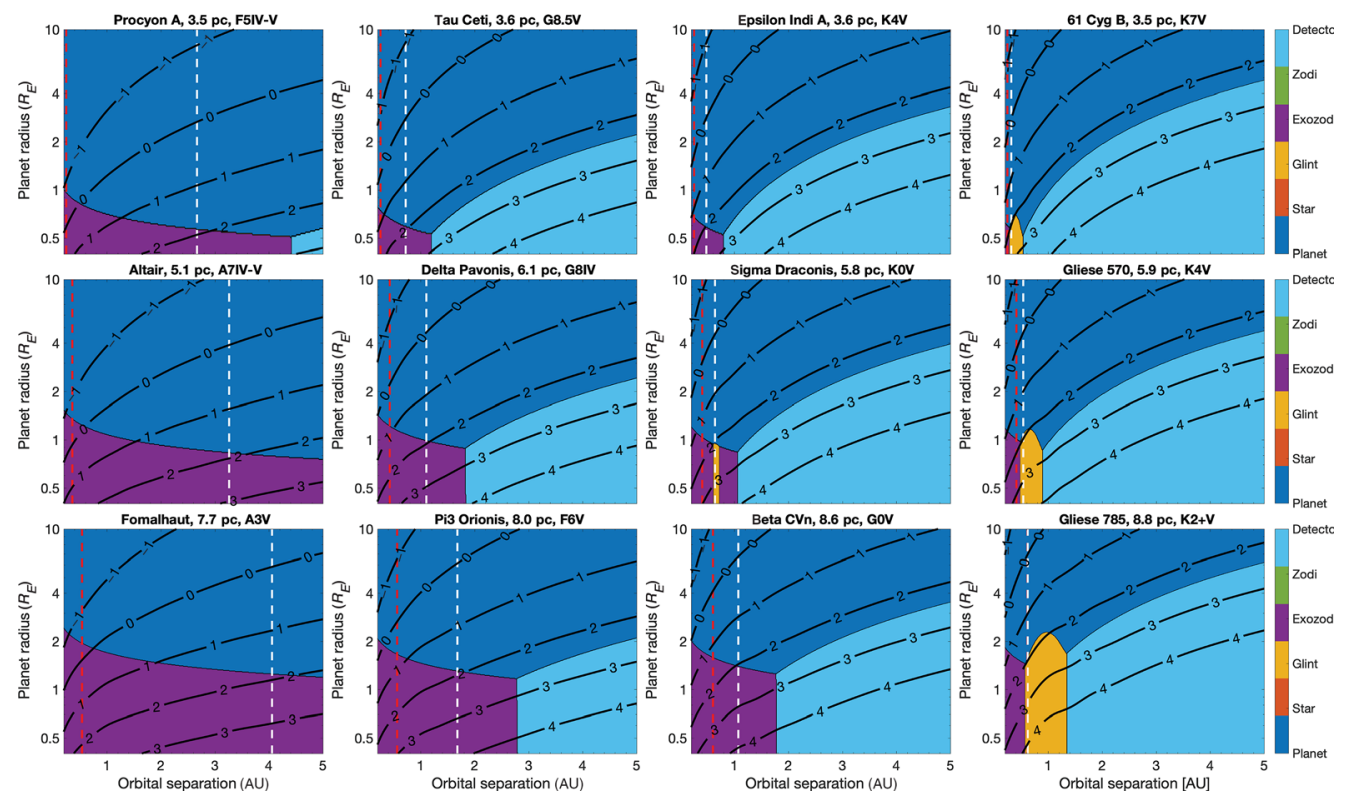

Fig. 8 Expected integration time $[S / N=20$ per spectral element, contour profiles, in log(hours)] and dominant noise source (shaded areas) expected in HabEx exploration of planets around nearby stars. The red dashed line corresponds to the IWA, and the blue dashed line corresponds to the planet-star separation for receiving Earth's insolation, i.e., the 1-AU equivalent. We adopt the nominal parameters in Table 1 in these simulations. With the additional optical edge coating, ${ }^{24}$ the impact of the solar glint would be eliminated.

We see that photon-limited detection and characterization of planets can be expected in the majority of the parameter space explored. In particular, HabEx could perform photon-limited planet characterization for Earth-sized planets in the HZs of nearby stars as far as $\sim 6$ parsecs. The integration time needed to achieve an $S / N=20$ spectrum at the resolution of $R=140$ is $\sim 10 \mathrm{~h}$ for the closest ( 3 to 4 parsecs) stars and 50 to $100 \mathrm{~h}$ for stars 5 to 6 parsecs away. These estimated integration times indicate more than one-order-of-magnitude improvement over the Starshade Rendezvous with Roman. The improvement comes from not only the larger telescope, but also higher throughput, better angular resolution to reduce the exozodiacal light interference, and lower solar glint brightness.

Exozodiacal light starts to affect the observations of Earth-sized planets around stars $\geq 8$ parsecs away. Figure 8 shows that the exozodiacal light is the dominant noise term for planets $<1.5 R_{\oplus}$ in the HZs of $\sim 8$-parsec stars. If the exozodiacal light can be subtracted from the photon-noise limit, however, the planets may still be observed and characterized with $S / N=$ 20 spectra within a few hundred hours. If the parameter $\alpha$ in Eq. (1) is further reduced from two to unity, the integration time in the exozodiacal-dominant regime would be smaller than what is shown in Fig. 8 by a factor $\sim 2$.

The potential targets for HabEx include more distant stars up to $\sim 15$ parsecs (Fig. 3). While not explicitly shown in Fig. 8, we estimate the impact of solar glint and exozodiacal light on the performance of planet observations around these farther stars. For the photometric aperture adopted in this study (i.e., of the diameter $\lambda / D$ ), we estimate that the exozodiacal flux from a " 3 zodis" disk has a magnitude of 28.3 at the HZ. The flux from the solar glint, with coating and $46 \%$ encircled energy, has a magnitude of 30.4 . The performance in each distance group is characterized by the latest-type (i.e., the least luminous) stars in that group, and the HZs of these stars typically have the angular separation corresponding to the IWA (70 mas). In the case of Gliese 785 , for example, the $\mathrm{HZ}$ is at $\sim 0.62 \mathrm{AU}$, and a $1-R_{\oplus}$ planet would have a planet-star contrast of $8.5 \times 10^{-10}$. With the star's visual magnitude of 6 and the encircled energy of $46 \%$ in the photometric aperture, the planet would have a magnitude 29.4. This is consistent with Fig. 8: a $1-R_{\oplus}$ planet would be brighter than the solar glint but less bright than the exozodiacal light, and a 1.6- $R_{\oplus}$ planet would be approximately as bright as the exozodiacal light. Applying the same analysis to a 15 -parsec star, the $\mathrm{HZ}$ is at $\sim 1.05 \mathrm{AU}$ (derived from the IWA), and a $1-R_{\oplus}$ planet 
would have a planet-star contrast of $2.6 \times 10^{-10}$. The star will have a similar apparent magnitude as Gliese 785 because its luminosity would scale as the square of the distance to keep the angular separation of the HZ at the IWA. The planet would then have a magnitude of 30.7 in the photometric aperture. This implies that, for the most distant stars in HabEx's planet search, a 1- $R_{\oplus}$ planet would be as bright as the solar glint and a $3-R_{\oplus}$ planet would be as bright as the exozodiacal light. As such, HabEx with coating generally does not have solar glint as the main noise term and is increasingly impacted by exozodiacal light for stars 8 to 15 parsecs away.

\section{Discussion}

\subsection{Requirements for Background Calibration}

To this point, we work on the assumption that the background can be removed by imaging processing to the photon-noise limit [i.e., $\beta=0$ in Eq. (1)]. Since the solar glint and the exozodiacal light dominate over the planetary light in the noise budget in substantial fractions of Roman's and HabEx's search spaces for small planets (Figs. 4, 5, and 8), the ability to calibrate these noise terms is important for delivering the science capability described in Sec. 4.

Here we first quantify the precision needed for background calibration. Figure 9 shows the ratio between the planetary flux and the background flux from solar glint and exozodiacal light [i.e., $N_{P} / \beta N_{B}$, or $K$ in Eq. (2)]. As discussed in Sec. 2.1, the maximum $S / N$ achievable is $N_{P} / \beta N_{B}$ when $\beta$ is non-zero. Therefore, the ratio shown in Fig. 9 divided by the desired $S / N$ (i.e., 20 in this work) is the maximum tolerable residual fraction $(\beta)$ of the background after calibration.

For the closest stars ( $<4$ parsecs), the flux from a $1-R_{\oplus}$ planet in the $\mathrm{HZ}$ is similar to the flux of the exozodiacal light and is greater than the flux of solar glint by more than one order of magnitude (Fig. 9). This means that the exozodiacal light must be calibrated to 5\% to allow an $S / N$ of 20 . For farther stellar systems (5 to 6 parsecs), the requirement for the calibration
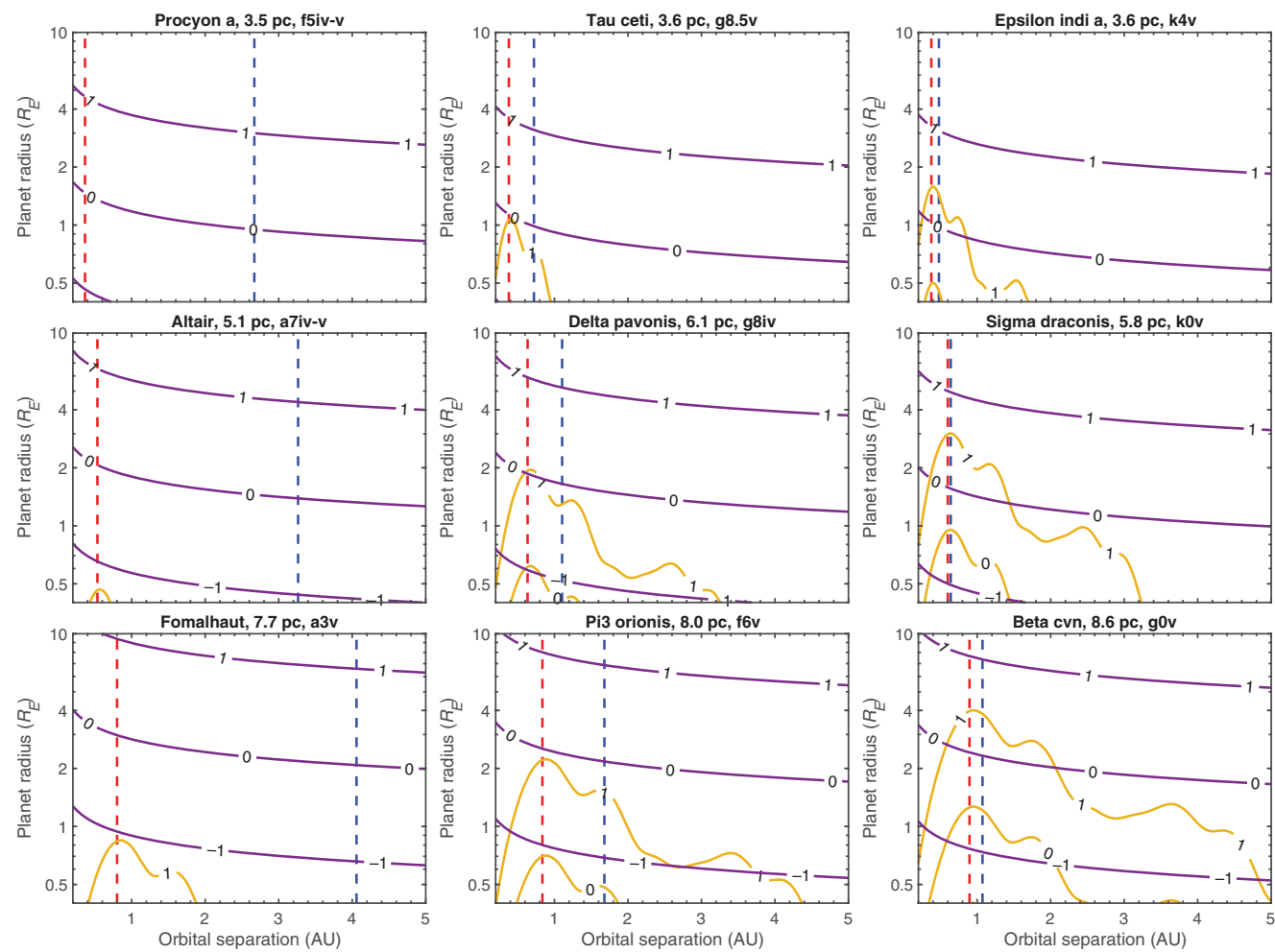

Fig. 9 The ratio between the planetary light and the solar glint (orange) and the ratio between the planetary light and the exozodiacal light (purple), for the same simulation parameters as Fig. 5. The base-10 logarithmic values are shown and labeled along the contour profiles. 
precision of exozodiacal light becomes $2 \%$, and that for the calibration of solar glint is $5 \%$. For stars $\sim 8$ parsecs away, the limiting science objective for a reasonable integration time with Roman is to characterize $1.5-R_{\oplus}$ planets in the HZs (Fig. 5). For this, the requirement for the calibration precision of exozodiacal light and solar glint remains $2 \%$ and $5 \%$, respectively. Taken together, the residual exozodiacal light should be $<2 \%$ (i.e., $\beta_{\text {exozodi }} \leq 0.02$ ) and the residual solar glint should be $<5 \%$ (i.e., $\beta_{\text {glint }} \leq 0.05$ ) to avoid significant adverse impact on the spectral characterization of small and temperate planets. At these critical precision levels of background calibration, the final $S / N$ is degraded from the $S / N$ with photon-noise-limit calibration by a factor of $\sqrt{2}$ [Eq. (2)], or $\sim 14$. To achieve a final $S / N$ of 20, the photon-noise-limit $S / N$ should be $20 \times \sqrt{2} \sim 28$; this is achievable by doubling the integration time from what is shown in Sec. 4 and better precision for background calibration (i.e., $\sim 1 \%$ for exozodiacal light).

Is this level of calibration achievable? For solar glint, which has a smooth intensity profile and only depends on the starshade position, orientation, and solar angle, standard image-processing techniques should be able to subtract it out with high precision. To enable this calibration, it may be necessary to carry out a reference observation of the solar glint after launch. A future community data challenge ${ }^{57}$ should develop and confirm the capability to subtract the solar glint with the help of the reference observation.

For exozodiacal light, the requirement for calibration is more stringent, and the ability to subtract and remove it depends on the spatial smoothness of the dust disk. One resolution element of Roman at $700 \mathrm{~nm}$ is $~ 60$ mas, which corresponds to 0.2 to $0.5 \mathrm{AU}$ in the nearby planetary systems. Therefore, density fluctuation of exozodiacal disks at this scale may adversely impact the calibration and extraction of the embedded planetary signal. Although there has not been a direct observation of the exozodiacal light in the HZs of nearby stars in the visible and near-infrared wavelengths, theoretical models of the origin of the dust particles may shed light on their distribution. In situ production of the dust by collisions and giant impacts leads to bright and localized dust "clumps," ${ }^{58}$ but these events are rare in mature planetary systems, which is the case for most stars in the target lists of Roman and HabEx. Dust particles may also be transported to the HZs by Poynting-Roberson drag ${ }^{59}$ or delivered as comets scattered by outer planets and then sublimated. ${ }^{60}$ The latter two processes eventually produce a smoothly varying dust density profile $^{61}$ and may enable high-precision background removal. However, dust particles may be trapped in mean-motion resonance with the planets ${ }^{62,63}$ and become "clumps." In addition to spatial structures, exozodiacal dust particles have very different spectral shapes than the planets, which may provide another way to distinguish them. We stress that, unlike other background terms, the exozodiacal signal, as well as the density profile that it implies, is a science objective in its own right. Its spatial distribution and origin would be studied together with the planets.

\subsection{Variability of the Background}

Another potential challenging aspect of background calibration is that the background may vary during the long integration typically required for planet characterization. As demonstrated in Sec. 4, the solar glint is the dominant background term compared with other stray light sources and residual starlight. The solar glint brightness is expected to vary as it depends on the lateral position of the starshade with respect to the line of sight. The next-in-the-line stray light source, reflection of earthshine and the Milky Way (Sec. 3.3), can be variable as well. ${ }^{64}$ The solar glint and other stray light to starshade exoplanet imaging is the analog of "speckles" to coronagraph exoplanet imaging, and the variability of speckles has driven the design of the coronagraph instrument on Roman. ${ }^{65}$ Here we provide a high-level estimate on the impact of background variability on starshade exoplanet imaging.

We approximate the background variability as a random variation, because the variation of the starshade's lateral position has a shorter timescale $\left(<10 \mathrm{~min}^{12}\right)$ compared with typical integration times. A change in the starshade's position is equivalent to a change in the off-axis angle for this problem, and the brightness of the residual starlight and the solar glint has a fractional change as a function of the off-axis angle (Fig. 2). We thus approximate the background flux with a $\log$-normal distribution with the parameter 0 and $\sigma[\log \operatorname{normal}(0, \sigma)]$ with respect to the median value. We perform Monte Carlo simulations of photons arriving onto two photometric apertures on the detector. Both apertures receive photons from the varying background, and one 

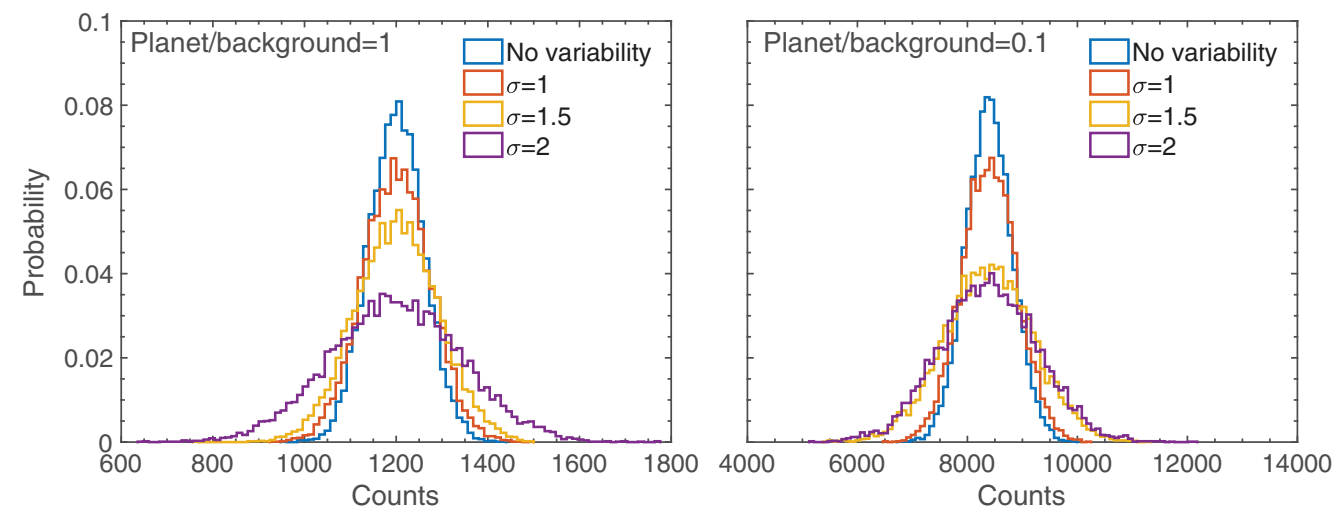

Fig. 10 Effect of background variability on image subtraction and planet detection. For each scenario, 10,000 instances of observations are simulated. Each observation consists of photometry of two apertures, one with the background and the other with the background and the planet, followed by subtraction of one aperture from the other. Each observation is divided into 100 segments, and the background flux in each segment varies around the median flux and follows a log-normal distribution with $\sigma$ shown in this figure. The counts of the apertures in each segment follow a Poisson distribution with a mean of the background flux of that segment (and the planet flux if any). The standard deviation of the resulting distribution characterizes the uncertainty of the planet flux measurement. In both cases, the median counts are chosen so that $S / N=20$ of the planet measurement is achieved at the limit of no variability.

of the pixels additionally receives photons from the planet. The count difference between the two apertures - as well as the statistical distribution of it - thus indicates how well the planet is detected. Figure 10 shows the resulting distributions for different levels of variability.

First, we see that the mean count in the subtracted aperture remains the same with and without the variability in Fig. 10. This indicates that the random variability does not bias the measurement of the planetary flux.

Figure 10 also shows that the resulting distribution becomes wider with greater background variability, indicating that background variability degrades the $S / N$ of the planet measurement. When the planetary flux is comparable to the background flux, the standard deviation of the resulting distribution increases from that of an invariant scenario by $11 \%, 62 \%$, and $106 \%$ when the value of $\sigma$ is $1,1.5$, and 2, respectively. If the planetary flux is one order of magnitude less than the background flux, the standard deviation increases by $22 \%, 91 \%$, and $167 \%$, respectively. Therefore, a random variability that follows a log-normal distribution up to $\sigma=1$ would not cause an appreciable change in the planet $S / N$, but a more variant background would significantly degrade the $S / N$, especially when the image is background dominated.

To put this into perspective, a log-normal distribution of $\sigma=1$ implies that the background flux stays within approximately half an order of magnitude from the median value in $68 \%$ of time during observation. This allowed range of variability corresponds to a variation of 20 mas in the off-axis angle (Fig. 2) or 2.5-m in the lateral displacement in the case of Starshade Rendezvous with Roman. The demonstrated capability of formation flying should be able to control the starshade to stay well within this range during science operation. ${ }^{12}$ The allowed range of variability (i.e., half an order of magnitude) appears to be also greater than the variability of earthshine. ${ }^{64}$ Therefore, the variation in the solar glint and residual starlight caused by the starshade's motion in formation flying, as well as the variation in the brightness of the earthshine, should not result in substantial degradation of the $S / N$ of planet measurements.

\subsection{Sensitivity of Exozodi Levels}

We have assumed the exozodi level to be 3 zodis in this work, guided by the best-fit median value from the hitherto most sensitive exozodiacal dust survey. ${ }^{25}$ However, the survey showed a large spread in the amount of exozodi light in nearby stars, with the $95 \%$ upper limit of 27 zodis. $^{25}$ With a larger exozodi level, observations in a greater search space of planetary size and orbital 
separation (Figs. 4, 5, and 8) are dominated by exozodiacal light. In the exozodi-dominated regime, the required integration time and the precision for background calibration increases linearly as the exozodi level increases [Eqs. (1) and (2)].

Of particular interest is where nearly photon-limited planetary spectroscopy may be expected with good background calibration. If the exozodi level increases from 3 to 10 zodis, the boundary between the planet-dominated regime and the exozodi-dominated regime increases from $\sim 1 R_{\oplus}$ to $\sim 1.8 R_{\oplus}$ for Roman observing the nearest ( $<4$ parsecs) stars (Fig. 5). The boundary increases to $>3 R_{\oplus}$ for farther stars. This means that an increase of the exozodi level could prevent Roman Rendezvous to perform photon-limited planetary spectroscopy for any target stars. HabEx on the other hand is less susceptible to high exozodi levels. If the exozodi level increases from 3 to 10 zodis, HabEx is still able to obtain photon-limited planetary spectroscopy for Earth-sized or larger rocky planets $\left(<1.7 R_{\oplus}\right)$ around stars $<6$ parsecs away (Fig. 8 ). The density and structure of the exozodiacal disks of nearby stars will continue to be an important area of exploration.

\subsection{Implications on Mission Designs}

The analyses presented in this paper assume that the observations of the planets take place at the orbital phase angle of $\pi / 3$. In reality, we do not know planets and their orbital elements of most target stars. The design of future missions using starshades must consider the "search completeness" that factors in the randomness of the observation epoch in planets' orbital revolution and visibility. Both Starshade Rendezvous with Roman and HabEx include an essentially blind search of planets around the nearby stars, ${ }^{6,7}$ and the search completeness of Earth-sized planets in the $\mathrm{HZ}$ was the driving factor of design and science cases.

However, the feasibility of spectral characterization shown in this paper paints a remarkably consistent picture with the search completeness, even though they are very different metrics. The search completeness primarily concerns the ability to detect planets in the broadband with several temporally spaced visits, whereas spectral characterization may entail a long integration performed at a single visit. Figure 5 shows that characterizing Earth-sized and temperate planets is feasible around the nearest ( 3 to 4 parsecs) stars and only marginally feasible around slightly farther (5 to 6 parsecs) stars for Starshade Rendezvous with Roman. This is fully consistent with the search completeness estimate in which the overall habitable-zone characterization completeness is $>25 \%$ for the nearest stars and $\sim 10 \%$ or less for the slightly farther stars. ${ }^{66}$ In other words, a more favorable target for spectral characterization shown in this paper generally has more favorable search completeness.

Interestingly, the nearest stars (procyon A, $\tau$ ceti, indi A, and sirius A, exhaustively) are truly the outstanding targets for both Starshade Rendezvous with Roman and HabEx for the highsearch completeness, relatively loose requirement of background calibration, and short integration time to characterize Earth-sized planets in their HZs. With a starshade, Roman can already measure spectra and characterize the atmospheres of Earth-sized planets of these stars. HabEx would further provide the wide spectral coverage to pinpoint atmospheric abundance and, with an integration of hours for each spectrum, the possibility of measuring the variability of the spectra. The spectral variability informs surface compositions (e.g., land and sea) and indicates variable cloud coverage and hydrological cycles ${ }^{64,67,68}$ - this feat is feasible only for the nearest stars using HabEx. Among the four stars, however, an outer dust disk has been detected with farinfrared and radio observations around $\tau$ ceti, ${ }^{52-54}$ and procyon A and sirius A have white dwarf companions, the evolution of which might have adversely impacted habitability of any planets nearby. This leaves $\epsilon$ indi A apparently as the most promising target. Because of this revelation, we suggest that mission designs should set a high priority to maximize the search completeness of planets in the HZs of these four stars, and particularly $\epsilon$ indi A, and encourage precursor efforts such as radial-velocity measurements that prioritize these stars.

We used the reference wavelength of $700 \mathrm{~nm}$ for the analyses presented in this work, and it provides a representation of the "green" band of Starshade Rendezvous with Roman (615 to $\left.800 \mathrm{~nm}^{66}\right)$ and the UV-visible band of HabEx $\left(0.3\right.$ to $\left.1.0 \mu \mathrm{m}^{7}\right)$. Roman may also have imaging and spectroscopy capabilities in the "blue" band ( 425 to $552 \mathrm{~nm}^{6}$ ). The blue band offers a few advantages over the green band, with less bright solar glint by 2 magnitudes ${ }^{11}$ and a smaller PSF and thus less exozodiacal light by a factor of $\sim 2$. These advantages reduce the needed integration 
time to achieve $S / N$ and loosen the requirements on background calibration. The blue band, however, requires a larger separation between the starshade and the telescope (37.2 Mm compared to $25.7 \mathrm{Mm}$ for the green band), leading to longer distances and higher fuel and time cost to maneuver the starshade from target to target. Also the blue band likely has fewer spectral features of interest from exoplanet atmospheres than the green band. ${ }^{44-49}$ In addition, HabEx performs near-infrared spectroscopy ( 1.0 to $1.8 \mu \mathrm{m}$ ) on selected "high-interest" targets. ${ }^{7}$ The magnitude of the solar glint has not been evaluated for the HabEx starshade in the near-infrared band, nor has the optical edge coating been designed to cover the near-infrared band. Future studies are required to quantify the solar glint and its impact on the science performance of HabEx in the 1.0- to $1.8-\mu \mathrm{m}$ band.

Finally, the planet search space that would be fully accessible for Starshade Rendezvous with Roman are large rocky planets $\left(\sim 1.5 R_{\oplus}\right)$ and potentially water worlds $\left(\sim 2.5 R_{\oplus}\right)$. For all stars in the target lists, these planets are likely within the reach from the perspectives of integration time and background calibration. The search completeness has not been assessed for these "superEarths," but we suspect that they would have reasonable completeness. Therefore, detailed designs of Starshade Rendezvous with Roman need to prioritize searching and characterizing large rocky planets and water worlds in the HZs, and relevant science investigations need to further quantify the requirements for their orbital determination and atmospheric abundance retrieval.

\section{Conclusion and Prospects}

We provided an overview and reassessment of the noise budget of exoplanet imaging and spectroscopy enabled by a starshade in formation flight with a space telescope. We developed a framework to estimate the $S / N$ of the planet observations of nearby stars using demonstrated performance parameters of starlight and stray light suppression resulting from S5 work. With an analysis of miscellaneous sources of stray light - from leakage through micrometeoroid damage to the reflection of earthshine-we show that the dominant stray light source is the scatter of sunlight by the edge of the starshade, i.e., the solar glint. The solar glint is a starshade-unique noise source and the starshade's analog to coronagraph speckles.

Applying our analysis framework to Starshade Rendezvous with Roman and HabEx, we find that starlight suppression delivered by the starshades is well enough to eliminate residual starlight from the dominant noise term, and the optical edge coating technology shown in Ref. 24 is necessary to prevent the solar glint from becoming the dominant noise. For a uniform dust level of 3 zodis, the dominant noise term is likely exozodiacal light for characterizing Earth-sized planets around stars $>4$ parsecs away with Roman and $>7$ parsecs away with HabEx. For closer stars, these missions with starshades would provide photon-limited measurements of Earth-sized planets if unbiased calibration of the background to the photon-noise limit can be achieved with reference observations and image processing.

Considering holistically the number of accessible stars, integration times, and demands for precise background calibration, one may expect Starshade Rendezvous with Roman to probe the nature of temperate and large rocky planets $\left(\sim 1.5 R_{\oplus}\right)$ and HabEx to study the nature of Earthsized planets and find true Earth twins. Based on the $S / N$ estimates presented in this paper, Roman with a starshade is capable of obtaining high-precision, moderate-resolution spectra of temperate and large rocky planets for stars as far as $\sim 8$ parsecs with a reasonable observation time, whereas spectroscopy of Earth-sized planets is likely limited to the nearest few stars. HabEx, with not only a larger telescope but also better angular resolution (to reduce exozodiacal light) and a more distant starshade (to reduce solar glint), would drastically reduce the required observation time and make it possible to characterize Earth-sized planets for stars $\sim 8$ parsecs and even farther away.

Based on the analyses presented here, we find it essential to validate the optical edge coating technology to eliminate the adverse impact of the solar glint on the science performance of Starshade Rendezvous with Roman and HabEx. Also as most scenarios of planet detection and characterization require precise calibration of the background, mostly from exozodiacal light, it is essential to develop the imaging processing techniques for the background calibration and 
validate their ability using realistic simulated images, for example, in a data challenge. ${ }^{57}$ In all, with unprecedented knowledge of starshade's optical performance and maturity of the associated technologies, we confirm that a starshade coupled with a sizeable space telescope continues to provide a near-term pathway toward finding habitable Earths in our interstellar neighborhood.

\section{Acknowledgments}

We thank Sergi Hildebrandt for providing example SISTER simulations and Karl Stapelfeldt, Eric Mamajek, and Andrew Romero-Wolf for helpful discussion. The research was carried out at the Jet Propulsion Laboratory, California Institute of Technology, under a contract with the National Aeronautics and Space Administration (No. 80NM0018D0004).

\section{References}

1. J. F. Kasting, D. P. Whitmire, and R. T. Reynolds, "Habitable zones around main sequence stars," Icarus 101(1), 108-128 (1993).

2. W. Cash, "Detection of earth-like planets around nearby stars using a petal-shaped occulter," Nature 442(7098), 51-53 (2006).

3. R. J. Vanderbei, E. Cady, and N. J. Kasdin, "Optimal occulter design for finding extrasolar planets," Astrophys. J. 665(1), 794 (2007).

4. D. Spergel et al., "Wide-field infrarred survey telescope-astrophysics focused telescope assets WFIRST-AFTA 2015 report," arXiv:1503.03757 (2015).

5. R. Akeson et al., "The wide field infrared survey telescope: 100 Hubbles for the 2020s," arXiv:1902.05569 (2019).

6. S. Seager et al., "Starshade rendezvous probe mission," in BAAS, Vol. 51, p. 106 (2019).

7. B. S. Gaudi et al., "The Habitable exoplanet observatory (HabEx) mission concept study final report," arXiv:2001.06683 (2020).

8. P. Willems et al., "Starshade to TRL5 (S5) Technology Development Plan," https:// exoplanets.nasa.gov/internal_resources/1033/ (2018).

9. A. Harness et al., "Demonstration of high contrast in monochromatic light at a flight-like Fresnel number," NASA Starshade Technology Development Activity Milestone Report 1a, 2019, https://exoplanets.nasa.gov/internal_resources/1210/.

10. A. Harness et al., "Demonstration of high contrast in broadband light at a flight-like Fresnel number," NASA Starshade Technology Development Activity Milestone Report 1b, 2019, https://exoplanets.nasa.gov/internal_resources/1211/.

11. E. Hilgemann et al., "Demonstration of solar glint lobe scatter performance," NASA Starshade Technology Development Activity Milestone Report 3, 2019, https://exoplanets .nasa.gov/internal_resources/1544/.

12. T. Flinois et al., "Lateral formation sensing and control," NASA Starshade Technology Development Activity Milestone Report 4, 2018, https://exoplanets.nasa.gov/internal_ resources/1077/.

13. M. C. Turnbull et al., "The search for habitable worlds. 1. The viability of a starshade mission," Publ. Astron. Soc. Pac. 124(915), 418 (2012).

14. C. C. Stark et al., "Maximized exoearth candidate yields for starshades," J. Astron. Telesc. Instrum. Syst. 2(4), 041204 (2016).

15. C. C. Stark et al., "Exoearth yield landscape for future direct imaging space telescopes," J. Astron. Telesc. Instrum. Syst. 5(2), 024009 (2019).

16. S. B. Shaklan et al., "Error budgets for the exoplanet starshade (Exo-S) probe-class mission study," Proc. SPIE 9605, $96050 \mathrm{Z}$ (2015).

17. C. C. Stark et al., "Maximizing the ExoEarth candidate yield from a future direct imaging mission," Astrophys. J. 795, 122 (2014).

18. T. P. Greene et al., "Characterizing transiting exoplanet atmospheres with JWST," Astrophys. J. 817(1), 17 (2016).

19. J. Krist et al., "Wfirst coronagraph flight performance modeling," Proc. SPIE 10698, 106982K (2018). 
20. S. R. Hildebrandt et al., "SISTER: Starshade Imaging Simulation Toolkit for Exoplanet Reconnaissance," J. Astron. Telesc. Instrum. Syst. (2021).

21. S. R. Hildebrandt et al., "Starshade Imaging Simulation Toolkit for Exoplanet Reconnaissance (SISTER," http://sister.caltech.edu (2019).

22. M. Kuchner, "Zodipic: zodiacal cloud image synthesis," Astrophysics Source Code Library, ascl-1202 (2012).

23. S. R. Martin et al., "Starshade optical edge modeling, requirements, and laboratory tests," Proc. SPIE 8864, 88641A (2013).

24. D. McKeithen et al., "Anti-reflection coatings on starshade optical edges for solar glint suppression," J. Astron. Telesc. Instrum. Syst. 7(2), 021208 (2021).

25. S. Ertel et al., "The HOSTS survey for exozodiacal dust: observational results from the complete survey," Astron. J. 159, 177 (2020).

26. S. Seager et al., "Exo-S: starshade probe-class exoplanet direct imaging mission concept final report," 2015, exep. jpl. nasa. gov/stdt.

27. G. Illingworth et al., "The HST extreme deep field (XDF): combining all ACS and WFC3/IR data on the HUDF region into the deepest field ever," Astrophys. J. Suppl. Ser. 209(1), 6 (2013).

28. E. Grün et al., "Orbital and physical characteristics of micrometeoroids in the inner solar system as observed by Helios 1," Planet. Space Sci. 28(3), 333-349 (1980).

29. B. G. Cour-Palais, "Space vehicle meteoroid shielding design," in ESA Comet Halley Micrometeoroid Hazard Workshop, Vol. 153, pp. 85-92 (1979).

30. F. Hörz, "Cratering and penetration experiments in aluminum and Teflon: implications for space-exposed surfaces," Meteorit. Planet. Sci. 47(4), 763-797 (2012).

31. W. P. Schonberg, "Debris cloud material characterization for hypervelocity impacts of single- and multimaterial projectiles on thin target plates," Shock Vib. 2(4), 273-287 (1995).

32. S. B. Shaklan et al., "Error budgeting and tolerancing of starshades for exoplanet detection," Proc. SPIE 7731, 77312G (2010).

33. A. Mallama and J. L. Hilton, "Computing apparent planetary magnitudes for the astronomical almanac," Astron. Comput. 25, 10-24 (2018).

34. B. Hoffmann et al., "Photographic surface photometry of the southern milky way-VIII. High-resolution and $r$ surface photometries of the southern milky way," Astron. Astrophys. Suppl. Ser. 128(3), 417-422 (1998).

35. B. J. Fulton and E. A. Petigura, "The California-Kepler survey. VII. Precise planet radii leveraging GAIA DR2 reveal the stellar mass dependence of the planet radius gap," Astron. J. 156(6), 264 (2018).

36. L. A. Rogers, "Most 1.6 earth-radius planets are not rocky," Astrophys. J. 801(1), 41 (2015).

37. J. E. Owen and Y. Wu, "The evaporation valley in the Kepler planets," Astrophys. J. 847(1), 29 (2017).

38. S. Jin and C. Mordasini, "Compositional imprints in density-distance-time: a rocky composition for close-in low-mass exoplanets from the location of the valley of evaporation," Astrophys. J. 853(2), 163 (2018).

39. L. Zeng et al., "Growth model interpretation of planet size distribution," Proc. Natl. Acad. Sci. U. S. A. 116(20), 9723-9728 (2019).

40. O. Mousis et al., "Irradiated ocean planets bridge super-earth and sub-Neptune populations," arXiv:2002.05243 (2020).

41. R. Pierrehumbert and E. Gaidos, "Hydrogen greenhouse planets beyond the habitable zone," Astrophys. J. Lett. 734(1), L13 (2011).

42. N. Madhusudhan et al., "The interior and atmosphere of the habitable-zone exoplanet k218b," Astrophys. J. Lett. 891(1), L7 (2020).

43. S. Seager, "Exoplanet habitability," Science 340(6132), 577-581 (2013).

44. R. E. Lupu et al., "Developing atmospheric retrieval methods for direct imaging spectroscopy of gas giants in reflected light. I. Methane abundances and basic cloud properties,' Astron. J. 152, 217 (2016).

45. M. Nayak et al., "Atmospheric retrieval for direct imaging spectroscopy of gas giants in reflected light. II. Orbital phase and planetary radius," Publ. Astron. Soc. Pac. 129, 034401 (2017). 
46. Y. K. Feng et al., "Characterizing earth analogs in reflected light: atmospheric retrieval studies for future space telescopes," Astron. J. 155, 200 (2018).

47. R. Hu, "Information in the reflected-light spectra of widely separated giant exoplanets," Astrophys. J. 887, 166 (2019).

48. M. Damiano and R. Hu, "Exorel: a Bayesian inverse retrieval framework for exoplanetary reflected light spectra," Astron. J. 159(4), 175 (2020).

49. M. Damiano, R. Hu, and S. R. Hildebrandt, "Multi-orbital-phase and multiband characterization of exoplanetary atmospheres with reflected light spectra," Astron. J. 160(5), 206 (2020).

50. M. Tuomi et al., "Signals embedded in the radial velocity noise-periodic variations in the $\tau$ ceti velocities," Astron. Astrophys. 551, A79 (2013).

51. F. Feng et al., "Color difference makes a difference: four planet candidates around $\tau$ ceti," Astron. J. 154(4), 135 (2017).

52. J. Greaves et al., "The debris disc around $\tau$ ceti: a massive analogue to the Kuiper belt," Mon. Not. R. Astron. Soc. 351(3), L54-L58 (2004).

53. S. Lawler et al., "The debris disc of solar analogue $\tau$ ceti: Herschel observations and dynamical simulations of the proposed multiplanet system," Mon. Not. R. Astron. Soc. 444(3), 2665-2675 (2014).

54. M. A. MacGregor et al., "Alma observations of the debris disk of solar analog $\tau$ ceti," Astrophys. J. 828(2), 113 (2016).

55. R. Hu et al., "The super-earth opportunity-search for habitable exoplanets in the 2020s," arXiv:1903.05258 (2019).

56. T. Glassman et al., "Starshade scaling relations," Proc. SPIE 7440, 744013 (2009).

57. R. Hu et al., "The Starshade exoplanet data challenge," J. Astron. Telesc. Instrum. Syst. (2021).

58. G. M. Kennedy and M. C. Wyatt, "The bright end of the exo-Zodi luminosity function: disc evolution and implications for exo-Earth detectability," Mon. Not. R. Astron. Soc. 433(3), 2334-2356 (2013).

59. G. M. Kennedy and A. Piette, "Warm exo-Zodi from cool exo-Kuiper belts: the significance of p-r drag and the inference of intervening planets," Mon. Not. R. Astron. Soc. 449(3), 2304-2311 (2015).

60. A. Bonsor and M. Wyatt, "The scattering of small bodies in planetary systems: constraints on the possible orbits of cometary material," Mon. Not. R. Astron. Soc. 420(4), 2990-3002 (2012).

61. A. Bonsor et al., "Using warm dust to constrain unseen planets," Mon. Not. R. Astron. Soc. 480(4), 5560-5579 (2018).

62. C. C. Stark and M. J. Kuchner, "The detectability of exo-Earths and super-earths via resonant signatures in exozodiacal clouds," Astrophys. J. 686(1), 637 (2008).

63. C. C. Stark, "The transit light curve of an exozodiacal dust cloud," Astron. J. 142(4), 123 (2011).

64. J. H. Jiang et al., "Using deep space climate observatory measurements to study the earth as an exoplanet," Astron. J. 156(1), 26 (2018).

65. B. Nemati, J. E. Krist, and B. Mennesson, "Sensitivity of the WFIRST coronagraph performance to key instrument parameters," Proc. SPIE 10400, 1040007 (2017).

66. A. Romero-Wolf et al., "Starshade rendezvous: exoplanet sensitivity and observing strategy," J. Astron. Telesc. Instrum. Syst. 7(2), 021210 (2021).

67. N. B. Cowan et al., "Alien maps of an ocean-bearing world," Astrophys. J. 700(2), 915 (2009).

68. Y. Fujii, E. L. Turner, and Y. Suto, "Variability of water and oxygen absorption bands in the disk-integrated spectra of earth," Astrophys. J. 765(2), 76 (2013).

Renyu Hu received his $\mathrm{PhD}$ in planetary science from Massachusetts Institute of Technology in 2013. He is a scientist at the Jet Propulsion Laboratory (JPL), and his research strives to identify and characterize habitable environments in the solar system and beyond. He is the starshade scientist of the NASA ExEP, providing science leadership to the S5 Starshade Technology Development Activity and managing the Starshade Science and Industry Partnership program. 
Doug Lisman received his BS degree in mechanical engineering from Washington University in St. Louis in 1984 and has been at the JPL since 1984. He is the systems engineering lead for Starshade Technology Development at the JPL, where he is a member of the Instrument Systems Engineering Group.

Stuart Shaklan received his $\mathrm{PhD}$ in optics from the University of Arizona in 1989 and has been at the JPL since 1991. He is the supervisor of the High Contrast Imaging Group in the Optics Section at the JPL.

Stefan Martin received his BSc degree in physics from the University of Bristol, United Kingdom, and his $\mathrm{PhD}$ in engineering from the University of Wales. He is a senior optical engineer at the JPL, where he has been leader of the TPF-I Flight Instrument Engineering Team, testbed lead for the TPF-I Planet Detection Testbed, and payload lead for the HabEx Telescope design study. He is currently involved in starshade accommodation on future space telescopes, such as NGRST.

Phil Willems received his BS degree in physics from the University of Wisconsin-Madison in 1988 and his $\mathrm{PhD}$ in physics from California Institute of Technology in 1997. He is an optical engineer at the JPL, where he is the manager of the S5 Starshade Technology Development Activity.

Kendra Short received her BS degree in mechanical and aerospace engineering from Princeton University and her MS degree in aero/astro engineering from Stanford University. She is currently the deputy flight system manager for NASA's Europa Clipper mission. Prior to this, she was the deputy program manager for the NASA ExEP, including S5 Starshade Technology Development Activity. 